\title{
STRINGY CHERN CLASSES OF SINGULAR TORIC VARIETIES AND THEIR APPLICATIONS
}

\author{
VICTOR BATYREV AND KARIN SCHALLER
}

\begin{abstract}
Let $X$ be a normal projective $\mathbb{Q}$-Gorenstein variety with at worst logterminal singularities. We prove a formula expressing the total stringy Chern class of a generic complete intersection in $X$ via the total stringy Chern class of $X$. This formula is motivated by its applications to mirror symmetry for Calabi-Yau complete intersections in toric varieties. We compute stringy Chern classes and give a combinatorial interpretation of the stringy Libgober-Wood identity for arbitrary projective $\mathbb{Q}$-Gorenstein toric varieties. As an application we derive a new combinatorial identity relating $d$-dimensional reflexive polytopes to the number 12 in dimension $d \geq 4$.
\end{abstract}

\section{INTRODUCTION}

The orbifold (or stringy) Euler number has been introduced by Dixon, Harvey, Vafa, and Witten [DHVW85] as a new topological invariant of singular varieties motivated by string theory. It was observed by Hirzebruch and Hofer [HH90] that the orbifold Euler number $e_{\text {orb }}(X)$ of a singular Calabi-Yau variety $X$ can be identified with the usual Euler number $e(Y)$ of its Calabi-Yau desingularization $Y$, i.e., a desingularization $\rho: Y \rightarrow X$ such that $Y$ is a smooth Calabi-Yau manifold. Such a desingularization $\rho$ might not be unique, but the Euler number $e(Y)$ does not depend on its choice. Moreover, the orbifold (or stringy) Hodge numbers $h_{\mathrm{str}}^{p, q}(X)$ of the singular Calabi-Yau variety $X$ can be defined in the same way as the Hodge numbers $h^{p, q}(Y)$ of the smooth Calabi-Yau manifold $Y$. The fact that the stringy Hodge numbers do not depend on the choice of a Calabi-Yau desingularization plays an important role in mirror symmetry for Calabi-Yau varieties [BD96].

More generally, a desingularization $\rho: Y \rightarrow X$ of a normal projective variety $X$ with at worst canonical Gorenstein singularities is called crepant if $\rho^{*} K_{X}=K_{Y}$. Using nonarchimedian integration Bat98, one can prove that the Euler number $e(Y)$ and the Hodge numbers $h^{p, q}(Y)$ do not depend on the choice of the crepant desingularization $\rho$. These numbers are called stringy Euler number $e_{s t r}(X)$ respectively stringy Hodge numbers $h_{s t r}^{p, q}(X)$ of the singular variety $X$.

It was discovered by Allufi Alu04 that not only the Euler number $e(Y)$ (the top Chern class $\left.c_{d}(Y)\right)$ of $Y$, but also the push-forwards of all other Chern classes $\rho_{*} c_{k}(Y) \in A^{k}(X)_{\mathbb{Q}}(0 \leq k \leq d)$ are independent of a crepant desingularization $\rho: Y \rightarrow X$ of a $d$-dimensional variety $X$. This observation led to the notion of stringy Chern classes $c_{k}^{\text {str }}(X) \in A^{k}(X)_{\mathbb{Q}}(0 \leq k \leq d)$ of singular varieties $X$. They have been introduced and developed in Alu05, dFLNU07. We remark that the 
stringy Chern class $c_{d-1}^{\text {str }}(X)$ of a singular $d$-dimensional projective variety $X$ appears in the stringy version of the Libgober-Wood formula [Bat00].

In this paper, we are interested in stringy Chern classes of generic complete intersections in toric varieties. Our interest is motivated by the well-known construction of many examples for Calabi-Yau varieties and their mirrors as hypersurfaces and complete intersections in Gorenstein toric Fano varieties [Bat94, BB97, BD96]. Another motivation for our paper was the search for a higher dimensional generalization of the well-known combinatorial identities for reflexive polytopes of dimension 2 and 3. This generalization and its connection to the Libgober-Wood formula for smooth manifolds [LW90] (see also [Sa196]) has been considered independently in the recent preprint of Godinho, von Heymann, and Sabatini [GvHS16]. Independently some results of our paper were obtained in the preprint of Douai [Dou16] motivated by Hertling's conjecture about the variance of the spectrum of tame regular functions.

Let $X$ be a $d$-dimensional normal projective $\mathbb{Q}$-Gorenstein variety with at worst log-terminal singularities, i.e., the canonical class $K_{X}$ of $X$ is a $\mathbb{Q}$-Cartier divisor and for some desingularization $\rho: Y \rightarrow X$ of $X$, whose exceptional locus is a union of smooth irreducible divisors $D_{1}, \ldots, D_{s}$ with only normal crossings, one has

$$
K_{Y}=\rho^{*} K_{X}+\sum_{i=1}^{s} a_{i} D_{i}
$$

for some rational numbers $a_{i}>-1(1 \leq i \leq s)$. The above desingularization $\rho$ will be called log-desingularization of $X$. For any nonempty subset $J \subseteq I:=\{1, \ldots, s\}$ we define $D_{J}$ to be the subvariety $\cap_{j \in J} D_{j}$ together with its closed embedding $e_{J}$ : $D_{J} \hookrightarrow Y$ and set $D_{\emptyset}:=Y$. We remark that the subvariety $D_{J} \subseteq Y$ is either empty or a smooth projective subvariety of $Y$ of codimension $|J|$.

If $V$ is an arbitrary smooth $d$-dimensional projective variety, then the E-polynomial of $V$ is defined as

$$
E(V ; u, v):=\sum_{0 \leq p, q \leq d}(-1)^{p+q} h^{p, q}(V) u^{p} v^{q}
$$

where $h^{p, q}(V)$ are the Hodge numbers of $V$. Furthermore, the Euler number $e(V)=$ $c_{d}(V)$ of $V$ equals $E(V ; 1,1)$. The stringy E-function of the singular variety $X$ is a rational algebraic function in two variables $u, v$ defined by the formula

$$
E_{s t r}(X ; u, v):=\sum_{\emptyset \subseteq J \subseteq I} E\left(D_{J} ; u, v\right) \prod_{j \in J}\left(\frac{u v-1}{(u v)^{a_{j}+1}-1}-1\right) .
$$

One can prove that the function $E_{s t r}(X ; u, v)$ does not depend on the choice of the log-desingularization $\rho$ Bat98, Theorem 3.4]. As a special case, this formula implies $E_{s t r}(X ; u, v)=E(Y ; u, v)$ if $\rho$ is a crepant desingularization of $X$. The top stringy Chern class (or the stringy Euler number) of $X$ is defined to be the limit of the stringy 
E-function (1), i.e.,

$$
c_{d}^{s t r}(X):=\lim _{u, v \rightarrow 1} E_{s t r}(X ; u, v)=\sum_{\emptyset \subseteq J \subseteq I} c_{d-|J|}\left(D_{J}\right) \prod_{j \in J}\left(\frac{-a_{j}}{a_{j}+1}\right),
$$

where $c_{d-|J|}\left(D_{J}\right)=e\left(D_{J}\right)$ denotes the Euler number of the smooth subvariety $D_{J} \subseteq$ $Y$. We regard the stringy top Chern class $c_{d}^{s t r}(X)$ as a special case of the $k$-th stringy Chern class defined in Alu05, dFLNU07 for any $k(0 \leq k \leq d)$. In this paper, we apply the following formula for the computation of the $k$-th stringy Chern class of a $d$-dimensional normal projective $\mathbb{Q}$-Gorenstein variety $X$ with at worst log-terminal singularities using the usual Chern classes of smooth projective subvarieties $D_{J} \subseteq Y$ :

$$
c_{k}^{s t r}(X):=\rho_{*}\left(\sum_{\emptyset \subseteq J \subseteq I} e_{J *} c_{k-|J|}\left(D_{J}\right) \prod_{j \in J}\left(\frac{-a_{j}}{a_{j}+1}\right)\right) \in A_{d-k}(X)_{\mathbb{Q}} .
$$

Here, $A_{d-k}(X)=A^{k}(X)$ denotes the Chow group of $(d-k)$-dimensional cycles on $X$ modulo rational equivalence and $A .(X)_{\mathbb{Q}}:=\bigoplus_{k=0}^{d} A_{d-k}(X)_{\mathbb{Q}}$ with $A_{d-k}(X)_{\mathbb{Q}}:=$ $A_{d-k}(X) \otimes \mathbb{Q}$ the rational Chow ring of $X$. Moreover, $\rho_{*}: A_{d-k}(Y) \rightarrow A_{d-k}(X)$ and $e_{J *}: A_{d-k}\left(D_{J}\right) \rightarrow A_{d-k}(Y)$ are push-forward homomorphisms corresponding to the proper birational morphism $\rho: Y \rightarrow X$ respectively the closed embeddings $e_{J}: D_{J} \hookrightarrow Y$. It is important to note that the above definition of stringy Chern classes is also independent of the log-desingularization $\rho$.

The paper is organized as follows:

In Section 1, we prove that the well-known formula expressing the total Chern class of smooth complete intersections via the total Chern class of the ambient smooth variety $V$ remains valid also for the total stringy Chern class $c_{\bullet}^{s t r}(\cdot)$ of generic hypersurfaces and complete intersections in the singular ambient variety $X$, i.e.,

$$
i_{*} c_{\bullet}^{s t r}\left(Z_{1} \cap \ldots \cap Z_{r}\right)=c_{\bullet}^{s t r}(X) \cdot \prod_{j=1}^{r}\left[Z_{j}\right]\left(1+\left[Z_{j}\right]\right)^{-1},
$$

where $Z_{1}, \ldots, Z_{r}$ are generic semiample Cartier divisors on $X$ and $i: Z_{1} \cap \ldots \cap Z_{r} \hookrightarrow X$ is the corresponding closed embedding. In particular, we show that the top stringy Chern class (or stringy Euler number) of generic semiample Cartier divisors $Z$ on $X$ can be computed via stringy Chern classes of $X$ by

$$
c_{d-1}^{s t r}(Z)=e_{s t r}(Z)=[Z] \cdot c_{d-1}^{s t r}(X)-[Z]^{2} \cdot c_{d-2}^{s t r}(X)+\ldots=\sum_{k=1}^{d}(-1)^{k-1}[Z]^{k} \cdot c_{d-k}^{s t r}(X) .
$$

We give a similar formula for the top stringy Chern class $c_{d-r}^{\text {str }}\left(Z_{1} \cap \ldots \cap Z_{r}\right)$ of complete intersections $Z_{1} \cap \ldots \cap Z_{r}$, where $Z_{1}, \ldots, Z_{r}$ are generic semiample Cartier divisors on the singular variety $X$.

In Section 2, we look at intersection numbers of stringy Chern classes with $\mathbb{Q}$ Cartier divisors. A particular case of such an intersection number has appeared in 
the stringy version of the Libgober-Wood identity

$$
\left.\frac{d^{2}}{d u^{2}} E_{s t r}(X ; u, 1)\right|_{u=1}=\frac{3 d^{2}-5 d}{12} c_{d}^{s t r}(X)+\frac{1}{6} c_{1}(X) \cdot c_{d-1}^{s t r}(X)
$$

in [Bat00, Theorem 3.8], where the intersection number $c_{1}(X) \cdot c_{d-1}^{s t r}(X)$ has been defined as

$$
c_{1}(X) \cdot c_{d-1}^{s t r}(X):=\sum_{\emptyset \subseteq J \subseteq I} \rho^{*} c_{1}(X) \cdot e_{J *} c_{d-|J|-1}\left(D_{J}\right) \prod_{j \in J}\left(\frac{-a_{j}}{a_{j}+1}\right)
$$

and its independence on the choice of the log-desingularization $\rho$ has been shown in [Bat00, Corollary 3.9]. In this paper, we consider more general intersection numbers $\left[Z_{1}\right] . \ldots .\left[Z_{k}\right] \cdot c_{d-k}^{s t r}(X)$, where $Z_{1}, \ldots, Z_{k}$ are arbitrary $\mathbb{Q}$-Cartier divisors. These intersection numbers can be defined by a similar formula

$$
\left[Z_{1}\right] \ldots \cdot\left[Z_{k}\right] \cdot c_{d-k}^{s t r}(X):=\sum_{\emptyset \subseteq J \subseteq I} \rho^{*}\left[Z_{1}\right] \ldots . \rho^{*}\left[Z_{k}\right] \cdot e_{J *} c_{d-|J|-k}\left(D_{J}\right) \prod_{j \in J}\left(\frac{-a_{j}}{a_{j}+1}\right) .
$$

We give a proof for its independence on the choice of $\rho$ without using the definition of stringy Chern classes.

In Section 3, we apply the results of the previous sections to hypersurfaces and complete intersections in normal projective $\mathbb{Q}$-Gorenstein toric varieties. For this purpose, we compute the $k$-th stringy Chern class of a $d$-dimensional projective $\mathbb{Q}$ Gorenstein toric variety $X$ associated with a fan $\Sigma$ of rational polyhedral cones in $N_{\mathbb{R}}$ as a linear combination of classes of torus-invariant cycles $X_{\sigma}$ corresponding to $k$-dimensional cones, i.e.,

$$
c_{k}^{s t r}(X)=\sum_{\sigma \in \Sigma(k)} v(\sigma) \cdot\left[X_{\sigma}\right]
$$

where $\Sigma(k)$ is the set of all $k$-dimensional cones of $\Sigma$ and $N_{\mathbb{R}}$ is a real vector space obtained by an extension of a $d$-dimensional lattice $N \cong \mathbb{Z}^{d}$. The coefficients $v(\sigma)$ are positive integers defined by the formula

$$
v(\sigma):=k ! \cdot \operatorname{vol}_{k}\left(\Theta_{\sigma}\right)
$$

where $\operatorname{vol}_{k}\left(\Theta_{\sigma}\right)$ is the $k$-dimensional volume of the lattice polytope $\Theta_{\sigma}$ obtained as the convex hull of the origin and the primitive lattice generators of all 1-dimensional faces of $\sigma$ with respect to the sublattice $\langle\sigma\rangle_{\mathbb{R}} \cap N$. We apply this formula for stringy Chern classes of toric varieties to compute intersection numbers $\left[D_{1}\right] . \ldots . .\left[D_{k}\right] . c_{d-k}^{s t r}(X)$ via mixed volumes of faces of divisor-associated convex lattice polytopes, where $D_{1}, \ldots, D_{k}$ are semiample torus-invariant $\mathbb{Q}$-Cartier divisors.

In Section 4, we are interested in a combinatorial interpretation of the stringy Libgober-Wood identity (3) for $d$-dimensional projective $\mathbb{Q}$-Gorenstein toric varieties. Such a toric variety $X$ is defined by a complete fan $\Sigma$ in $N_{\mathbb{R}}$ such that there exists a piecewise linear function $\kappa: N_{\mathbb{R}} \rightarrow \mathbb{R}$ that is linear on each cone $\sigma$ of $\Sigma$ and has value 
-1 on every primitive lattice generator of a 1-dimensional cone of $\Sigma$. A projective $\mathbb{Q}$ Gorenstein toric variety $X$ is called log-Fano toric variety if its anticanonical divisor $-K_{X}$ is ample, i.e., if $\kappa$ is strictly convex. In this case

$$
\Delta:=\left\{x \in N_{\mathbb{R}} \mid \kappa(x) \geq-1\right\}
$$

is a convex lattice polytope whose vertices are primitive lattice generators of 1 dimensional cones of $\Sigma$. Let $M:=\operatorname{Hom}(N, \mathbb{Z})$ be the dual lattice to $N$ and $\langle\cdot, \cdot\rangle$ : $M \times N \rightarrow \mathbb{Z}$ the natural pairing, which extends to a pairing $\langle\cdot, \cdot\rangle: M_{\mathbb{R}} \times N_{\mathbb{R}} \rightarrow \mathbb{R}$. The dual polytope $\Delta^{*}$ of $\Delta$ is a convex polytope with rational vertices defined as

$$
\Delta^{*}:=\left\{y \in M_{\mathbb{R}} \mid\langle y, x\rangle \geq-1 \forall x \in \Delta\right\} .
$$

The polytope $\Delta$ is called reflexive if all vertices of the dual polytope $\Delta^{*}$ belong to the lattice $M$. The latter case happens if and only if the log-Fano toric variety $X$ is a Gorenstein toric Fano variety (i.e., its anticanonical divisor is an ample Cartier divisor). Gorenstein toric Fano varieties $X$ of dimension $d$ associated with reflexive polytopes $\Delta$ are used in mirror symmetry as ambient spaces for Calabi-Yau hypersurfaces [Bat94, BB97, BD96]. The stringy Euler number of a generic ample Calabi-Yau hypersurface $Z$ in $X$ is combinatorially computable via

$$
e_{s t r}(Z)=c_{d-1}^{s t r}(Z)=\sum_{k=0}^{d-3}(-1)^{k} \sum_{\substack{\theta \leq \Delta \\ \operatorname{dim}(\theta)=k+1}} v(\theta) \cdot v\left(\theta^{*}\right),
$$

where the face $\theta^{*}:=\left\{y \in \Delta^{*} \mid\langle y, x\rangle=-1 \forall x \in \theta\right\}$ of the dual reflexive polytope $\Delta^{*}$ is called dual face to a face $\theta$ of $\Delta$ (written $\theta \preceq \Delta$ ).

If $X$ is a toric log del Pezzo surface associated to a convex lattice polytope $\Delta$ (cf. LDP-polytope [KKN10]), then the stringy Libgober-Wood identity (3) for $X$ is equivalent to the combinatorial equality

$$
v(\Delta)+v\left(\Delta^{*}\right)=12 \sum_{n \in \Delta \cap N}(\kappa(n)+1)^{2} .
$$

In particular, one always has $v(\Delta)+v\left(\Delta^{*}\right) \geq 12$ and equality holds, i.e.,

$$
v(\Delta)+v\left(\Delta^{*}\right)=12,
$$

if and only if $\Delta$ is a reflexive polytope.

If $\Delta$ is a 3-dimensional reflexive polytope, then the stringy Euler number of a Calabi-Yau hypersurface in the associated Gorenstein toric variety $X$ is 24 and one obtains the identity

$$
\sum_{\substack{\theta \leq \Delta \\ \operatorname{dim}(\theta)=1}} v(\theta) \cdot v\left(\theta^{*}\right)=24
$$

Our aim in Section 5 is to give a generalization of the above identities for reflexive polytopes of dimension $d \geq 4$. For this purpose, we use the Ehrhart power series of 
an arbitrary $d$-dimensional lattice polytope $\Delta \subseteq N_{\mathbb{R}}$ defined as

$$
P_{\Delta}(t):=\sum_{k \geq 0}|k \Delta \cap N| t^{k},
$$

where $|k \Delta \cap N|$ denotes the number of lattice points in $k \Delta$. This series can be written as the rational function

$$
P_{\Delta}(t)=\frac{\psi_{d}(\Delta) t^{d}+\ldots+\psi_{1}(\Delta) t+\psi_{0}(\Delta)}{(1-t)^{d+1}},
$$

where $\psi_{\alpha}(\Delta)$ are nonnegative integers for all $0 \leq \alpha \leq d$ [Bat93, Theorem 2.11]. We show that the stringy Libgober-Wood identity (3) for a Gorenstein toric Fano variety defined by the fan of cones over faces of a reflexive polytope $\Delta$ is equivalent to the combinatorial identity

$$
\sum_{\alpha \in[0, d] \cap \mathbb{Z}} \psi_{\alpha}(\Delta)\left(\alpha-\frac{d}{2}\right)^{2}=\frac{d}{12} v(\Delta)+\frac{1}{6} \sum_{\substack{\theta \preceq \Delta \\ \operatorname{dim}(\theta)=d-2}} v(\theta) \cdot v\left(\theta^{*}\right) .
$$

For reflexive polytopes $\Delta$ of dimension 2 and 3 this identity is equivalent to Equation (5) respectively (6), but for reflexive polytopes $\Delta$ of dimension $d \geq 4$ Equation (7) is not anymore symmetric with respect to the polar duality between $\Delta$ and $\Delta^{*}$ (i.e., the equality for $\Delta$ is not equivalent to the one for $\Delta^{*}$ ). If $d=4$, then (7) is equivalent to

$$
12 \cdot|\partial \Delta \cap N|=2 \cdot v(\Delta)+\sum_{\substack{\theta \leq \Delta \\ \operatorname{dim}(\theta)=2}} v(\theta) \cdot v\left(\theta^{*}\right),
$$

where $\partial \Delta$ denotes the boundary of the polytope $\Delta$ and $|\partial \Delta \cap N|$ the number of lattice points in $\partial \Delta$. In addition, we consider some generalizations of the above identities for Gorenstein polytopes.

\section{Stringy Chern Classes of Complete intersections}

First, we note that for projective varieties $X$ the general definition of the total stringy Chern class $c_{\bullet}^{s t r}(X) \in A_{\bullet}(X)_{\mathbb{Q}}$ due to de Fernex, Luperico, Nevins, and Uribe dFLNU07] can be simplified as follows:

Proposition 1.1. Let $X$ be a d-dimensional normal projective $\mathbb{Q}$-Gorenstein variety with at worst log-terminal singularities and $\rho: Y \rightarrow X$ a log-desingularization of $X$. Then the total stringy Chern class of $X$ can be computed via total Chern classes c. $\left(D_{J}\right)$ of smooth projective subvarieties $D_{J} \subseteq Y$ by

$$
c_{\bullet}^{s t r}(X):=\rho_{*}\left(\sum_{\emptyset \subseteq J \subseteq I} e_{J *} c_{\bullet}\left(D_{J}\right) \prod_{j \in J}\left(\frac{-a_{j}}{a_{j}+1}\right)\right) \in A \cdot(X)_{\mathbb{Q}} .
$$

In particular, one obtains

$$
c_{k}^{s t r}(X):=\rho_{*}\left(\sum_{\emptyset \subseteq J \subseteq I} e_{J *} c_{k-|J|}\left(D_{J}\right) \prod_{j \in J}\left(\frac{-a_{j}}{a_{j}+1}\right)\right) \in A_{d-k}(X)_{\mathbb{Q}}
$$


and has

$$
c_{k}^{s t r}(X)=\rho_{*} c_{k}(Y) \in A_{d-k}(X)_{\mathbb{Q}}
$$

if $\rho: Y \rightarrow X$ is a crepant log-desingularization $(0 \leq k \leq d)$.

Proof. The definition of the total stringy Chern class given by de Fernex, Luperico, Nevins, and Uribe in dFLNU07] uses the group homomorphism of MacPherson $c$ : $F(Y) \rightarrow A$. (Y) from the group $F(Y)$ of constructible functions on $Y$ to the Chow group $A_{\text {. }}(Y)$ of $Y$. If $\mathbb{1}_{Y}$ is the characteristic function of the smooth variety $Y$, then $c\left(\mathbb{1}_{Y}\right)=c_{\bullet}(Y)$. Using the stratification of $Y$ by locally closed subsets $D_{J}^{\circ}:=$ $D_{J} \backslash\left(\cup_{i \in I \backslash J} D_{i}\right)(\emptyset \subseteq J \subseteq I)$ their definition looks as follows:

$$
c_{\bullet}^{s t r}(X):=\rho_{*}\left(\sum_{\emptyset \subseteq J \subseteq I} c\left(\mathbb{1}_{D_{J}^{\circ}}\right) \prod_{j \in J}\left(\frac{1}{a_{j}+1}\right)\right) .
$$

Using the stratification $\mathbb{1}_{D_{J}}=\sum_{J^{\prime} \supseteq J} \mathbb{1}_{D_{J^{\prime}}^{\circ}}$ and $c\left(\mathbb{1}_{D_{J}}\right)=\sum_{J^{\prime} \supseteq J} c\left(\mathbb{1}_{D_{J^{\prime}}^{\circ}}\right)$ for all $\emptyset \subseteq J \subseteq I$, we conclude

$$
\begin{aligned}
\sum_{\emptyset \subseteq J \subseteq I} c\left(\mathbb{1}_{D_{J}^{\circ}}\right) \prod_{j \in J}\left(\frac{1}{a_{j}+1}\right) & =\sum_{\emptyset \subseteq J \subseteq I}\left(\sum_{J^{\prime} \supseteq J} c\left(\mathbb{1}_{D_{J^{\prime}}^{\circ}}\right)\right) \prod_{j \in J}\left(\frac{1}{a_{j}+1}-1\right) \\
& =\sum_{\emptyset \subseteq J \subseteq I} c\left(\mathbb{1}_{D_{J}}\right) \prod_{j \in J}\left(\frac{-a_{j}}{a_{j}+1}\right) .
\end{aligned}
$$

It remains to apply $\rho_{*}$ to the above equality and the property $c\left(\mathbb{1}_{D_{J}}\right)=e_{J_{*}} c \cdot\left(D_{J}\right)$, which follows from the commutative diagram

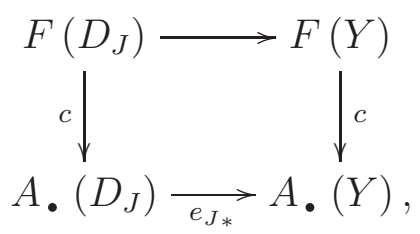

where $e_{J_{*}}: A_{\bullet}\left(D_{J}\right) \rightarrow A_{\bullet}(Y)$ is the push-forward homomorphism corresponding to the closed embedding $e_{J}: D_{J} \hookrightarrow Y$.

Remark 1.2. The important ingredient in the definition of the total stringy Chern $\operatorname{class} c_{\bullet}^{\text {str }}(X)$ (resp. $k$-th stringy Chern class $c_{k}^{s t r}(X)$ ) is its independence on the choice of the log-desingularization $\rho$. This property was proved in dFLNU07, Proposition $3.2]$.

Let $V$ be a smooth $d$-dimensional normal projective variety and $Z$ a smooth divisor on $V$ with the closed embedding $i: Z \hookrightarrow V$. Using the exact sequence of vector bundles $0 \rightarrow \mathcal{T}_{Z} \rightarrow i^{*} \mathcal{T}_{V} \rightarrow \mathcal{O}_{Z}(Z) \rightarrow 0$, one obtains a formula that computes the 
total Chern class of $Z$ in terms of the total Chern class of the ambient variety $V$ :

$$
i_{*} c_{\bullet}(Z)=c_{\bullet}(V) \cdot[Z](1+[Z])^{-1}=c_{\bullet}(V) \cdot\left(\sum_{k=1}^{\infty}(-1)^{k-1}[Z]^{k}\right),
$$

where $\mathcal{T}$. denotes the tangent bundle, $[Z]$ the class of $Z$ in $A_{d-1}(V)$, and $c_{\bullet}(V):=$ $\sum_{k} c_{k}(V)$ the total Chern class of $V$ (cf. [Ful98, Example 3.2.12]). In particular, the Euler number of $Z$ can be computed as

$$
e(Z)=c_{d-1}(Z)=\sum_{k=1}^{d}(-1)^{k-1}[Z]^{k} \cdot c_{d-k}(V) .
$$

We show that the same formulas hold for the total stringy Chern class respectively the top stringy Chern class of generic hypersurfaces in singular varieties.

Theorem 1.3. Let $X$ be a normal projective $\mathbb{Q}$-Gorenstein variety with at worst logterminal singularities and $Z$ a generic semiample Cartier divisor on $X$. Then the total stringy Chern class of $Z$ is

$$
i_{*} c_{\bullet}^{s t r}(Z)=c_{\bullet}^{s t r}(X) \cdot[Z](1+[Z])^{-1},
$$

where $i: Z \hookrightarrow X$ is the closed embedding.

Proof. Let $\rho: Y \rightarrow X$ be a log-desingularization of $X$ and $K_{Y}=\rho^{*} K_{X}+\sum_{i=1}^{s} a_{i} D_{i}$. By Theorem of Bertini, $Z^{\prime}:=\rho^{-1}(Z)$ is a smooth divisor on $Y$. By the adjunction formula $K_{Z^{\prime}}=\left.\left(K_{Y}+Z^{\prime}\right)\right|_{Z^{\prime}}$, we obtain $K_{Z^{\prime}}=\rho^{*} K_{Z}+\sum_{i=1}^{s} a_{i} D_{i}^{\prime}$, where $D_{i}^{\prime}:=D_{i} \cap Z^{\prime}$. Define $D_{J}^{\prime}:=\cap_{j \in J} D_{j}^{\prime}$ and note that $D_{J}^{\prime}=D_{J} \cap Z^{\prime} \subseteq D_{J}$ is a smooth divisor on $D_{J}$. Let $e_{J *}^{\prime}: A_{\bullet}\left(D_{J}^{\prime}\right) \rightarrow A_{\bullet}\left(Z^{\prime}\right)$ be the push-forward homomorphism corresponding to the closed embedding $e_{J}^{\prime}: D_{J}^{\prime} \hookrightarrow Z^{\prime}$. Consider the commutative diagram

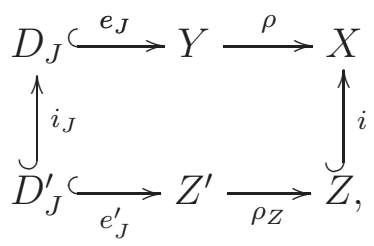

where $\rho_{Z}: Z^{\prime} \rightarrow Z$ and $i_{J}: D_{J}^{\prime} \rightarrow D_{J}$ are restrictions of $\rho$ respectively $i$. We apply Equation (11) to the smooth divisor $D_{J}^{\prime} \subseteq D_{J}$ and obtain

$$
i_{*} \rho_{Z *} e_{J *}^{\prime} c_{\bullet}\left(D_{J}^{\prime}\right)=\rho_{*} e_{J *} i_{J *} c_{\bullet}\left(D_{J}^{\prime}\right)=\rho_{*} e_{J *}\left(c \cdot\left(D_{J}\right) \cdot\left[D_{J}^{\prime}\right]\left(1+\left[D_{J}^{\prime}\right]\right)^{-1}\right) .
$$

Using the projection formula twice provides

$$
e_{J *}\left(c \cdot\left(D_{J}\right) \cdot\left[D_{J}^{\prime}\right]\left(1+\left[D_{J}^{\prime}\right]\right)^{-1}\right)=e_{J *} c_{\bullet}\left(D_{J}\right) \cdot\left[Z^{\prime}\right]\left(1+\left[Z^{\prime}\right]\right)^{-1}
$$

and

$$
\rho_{*}\left(e_{J *} c \cdot\left(D_{J}\right) \cdot\left[Z^{\prime}\right]\left(1+\left[Z^{\prime}\right]\right)^{-1}\right)=\rho_{*} e_{J *} c \cdot\left(D_{J}\right) \cdot[Z](1+[Z])^{-1}
$$


because $e_{J}^{*} Z^{\prime}=D_{J}^{\prime}, \rho^{*} Z=Z^{\prime}$ and the pull-backs $e_{J}^{*}, \rho^{*}$ are homomorphisms. Therefore, we get

$$
i_{*} \rho_{*} e_{J *}^{\prime} c_{\bullet}\left(D_{J}^{\prime}\right)=\rho_{*} e_{J *} c \cdot\left(D_{J}\right) \cdot[Z](1+[Z])^{-1} .
$$

By applying Proposition 1.1 to $Z$ and $X$, we conclude

$$
\begin{aligned}
i_{*} c_{\bullet}^{s t r}(Z) & =i_{*} \rho_{*}\left(\sum_{\emptyset \subseteq J \subseteq I} e_{J *}^{\prime}\left(c \cdot\left(D_{J}^{\prime}\right) \prod_{j \in J}\left(\frac{-a_{j}}{a_{j}+1}\right)\right)\right) \\
& =\rho_{*}\left(\sum_{\emptyset \subseteq J \subseteq I} e_{J *} c_{\bullet}\left(D_{J}\right) \prod_{j \in J}\left(\frac{-a_{j}}{a_{j}+1}\right)\right) \cdot[Z](1+[Z])^{-1} \\
& =c_{\bullet}^{s t r}(X) \cdot[Z](1+[Z])^{-1} .
\end{aligned}
$$

Corollary 1.4. Let $X$ be a normal projective $\mathbb{Q}$-Gorenstein variety of dimension $d$ with at worst log-terminal singularities and $Z$ a generic semiample Cartier divisor on $X$. Then the stringy Euler number of $Z$ is

$$
e_{s t r}(Z)=c_{d-1}^{s t r}(Z)=\sum_{k=1}^{d}(-1)^{k-1}[Z]^{k} \cdot c_{d-k}^{s t r}(X) .
$$

If $[Z]=c_{1}(X), Z$ has trivial anticanonical class and the formula simplifies to

$$
e_{s t r}(Z)=c_{d-1}^{s t r}(Z)=\sum_{k=1}^{d-2}(-1)^{k-1}[Z]^{k} \cdot c_{d-k}^{s t r}(X) .
$$

The formulas in Theorem 1.3 and Corollary 1.4 for total stringy Chern classes respectively top stringy Chern classes of generic hypersurfaces can be generalized to generic complete intersections in singular varieties:

Theorem 1.5. Let $X$ be a normal projective $\mathbb{Q}$-Gorenstein variety with at worst log-terminal singularities, $Z_{1}, \ldots, Z_{r}$ generic semiample Cartier divisors on $X$, and $i: Z_{1} \cap \ldots \cap Z_{r} \hookrightarrow X$ the closed embedding. Then the total stringy Chern class of the complete intersection $Z_{1} \cap \ldots \cap Z_{r}$ is

$$
i_{*} c_{\bullet}^{s t r}\left(Z_{1} \cap \ldots \cap Z_{r}\right)=c_{\bullet}^{s t r}(X) . \prod_{j=1}^{r}\left[Z_{j}\right]\left(1+\left[Z_{j}\right]\right)^{-1} .
$$

Proof. We apply induction on $r$ and use Theorem $1.3 r$-times, since for any $2 \leq r^{\prime} \leq r$ the complete intersection $Z_{1} \cap \ldots \cap Z_{r^{\prime}}$ is a generic hypersurface in $Z_{1} \cap \ldots \cap Z_{r^{\prime}-1}$.

Corollary 1.6. Let $X$ be a normal projective $\mathbb{Q}$-Gorenstein variety of dimension $d$ with at worst log-terminal singularities and $Z_{1}, \ldots, Z_{r}$ generic semiample Cartier divisors on $X$. Then the stringy Euler number $c_{d-r}^{\text {str }}\left(Z_{1} \cap \ldots \cap Z_{r}\right)$ of the complete 
intersection $Z_{1} \cap \ldots \cap Z_{r}$ is

$$
\sum_{k=0}^{d-r}(-1)^{k}\left[Z_{1}\right] \ldots \cdot\left[Z_{r}\right] \cdot\left(\sum_{\substack{j_{0}, \ldots, j_{k}=1 \\ j_{0} \leq \ldots \leq j_{k}}}^{r}\left[Z_{j_{0}}\right] \ldots \cdot\left[Z_{j_{k}}\right]\right) \cdot c_{d-r-k}^{s t r}(X) .
$$

Corollary 1.7. Let $X$ be a normal projective $\mathbb{Q}$-Gorenstein variety of dimension $d$ with at worst log-terminal singularities and $Z_{1}, \ldots, Z_{r}$ generic semiample Cartier divisors on $X$ such that $[Z]:=\left[Z_{1}\right]=\ldots=\left[Z_{r}\right]$. Then the stringy Euler number $c_{d-r}^{s t r}\left(Z_{1} \cap \ldots \cap Z_{r}\right)$ of the complete intersection $Z_{1} \cap \ldots \cap Z_{r}$ is

$$
c_{d-r}^{s t r}\left(Z_{1} \cap \ldots \cap Z_{r}\right)=\sum_{k=0}^{d-r}(-1)^{k}\left(\begin{array}{c}
k+r-1 \\
r-1
\end{array}\right)[Z]^{r+k} \cdot c_{d-r-k}^{s t r}(X) .
$$

\section{InterseCtion NUMBers With STRINGy CHERn Classes}

Let $X$ be a $d$-dimensional normal projective $\mathbb{Q}$-Gorenstein variety with at worst log-terminal singularities, $\rho: Y \rightarrow X$ a log-desingularization of $X$, and $Z_{1}, \ldots, Z_{k}$ arbitrary $\mathbb{Q}$-Cartier divisors on $X$. Intersecting the classes $\left[Z_{1}\right], \ldots,\left[Z_{k}\right] \in \operatorname{Pic}(X)_{\mathbb{Q}}$ with the stringy Chern class $c_{d-k}^{s t r}(X) \in A_{k}(X)_{\mathbb{Q}}$, one obtains the rational intersection number $\left[Z_{1}\right] \ldots .\left[Z_{k}\right] \cdot c_{d-k}^{s t r}(X)$, which can be considered as a generalization of Equation (4). Using (9) for the definition of the stringy Chern class $c_{d-k}^{s t r}(X) \in A_{k}(X)_{\mathbb{Q}}$ and the projection formula for the proper morphism $\rho$, one receives

$$
\begin{aligned}
{\left[Z_{1}\right] \ldots . \ldots\left[Z_{k}\right] \cdot c_{d-k}^{s t r}(X) } & \left.=\left[Z_{1}\right] \ldots \cdot \ldots Z_{k}\right] \cdot \rho_{*}\left(\sum_{\emptyset \subseteq J \subseteq I} e_{J *} c_{d-|J|-k}\left(D_{J}\right) \prod_{j \in J}\left(\frac{-a_{j}}{a_{j}+1}\right)\right) \\
& \left.=\rho_{*}\left(\rho^{*}\left(\left[Z_{1}\right] \ldots \cdot \ldots Z_{k}\right]\right) \cdot \sum_{\emptyset \subseteq J \subseteq I} e_{J *} c_{d-|J|-k}\left(D_{J}\right) \prod_{j \in J}\left(\frac{-a_{j}}{a_{j}+1}\right)\right) \\
& =\sum_{\emptyset \subseteq J \subseteq I} \rho^{*}\left[Z_{1}\right] \ldots \rho^{*}\left[Z_{k}\right] \cdot e_{J *} c_{d-|J|-k}\left(D_{J}\right) \prod_{j \in J}\left(\frac{-a_{j}}{a_{j}+1}\right),
\end{aligned}
$$

where the homomorphism $\rho^{*}: \operatorname{Pic}(X)_{\mathbb{Q}} \rightarrow \operatorname{Pic}(Y)_{\mathbb{Q}}$ is determined by the pullback of line bundles and the intersection product

$$
\rho^{*}\left[Z_{1}\right] . \ldots . \rho^{*}\left[Z_{k}\right] . e_{J *} c_{d-|J|-k}\left(D_{J}\right)
$$

is the value of the multilinear map $\operatorname{Pic}(Y)_{\mathbb{Q}}^{k} \times A_{k}(Y) \rightarrow \mathbb{Q}$ defined by the intersection of $k$ classes $\rho^{*}\left[Z_{1}\right], \ldots, \rho^{*}\left[Z_{k}\right]$ of $\mathbb{Q}$-Cartier divisors on $Y$ with the $k$-dimensional cycle $e_{J *} c_{d-|J|-k}\left(D_{J}\right) \in A_{k}(Y)$ composed with the natural map $A_{0}(Y)_{\mathbb{Q}} \rightarrow \mathbb{Q}$. In particular, we obtain

Theorem 2.1. The rational intersection number

$$
\sum_{\emptyset \subseteq J \subseteq I} \rho^{*}\left[Z_{1}\right] \ldots . \rho^{*}\left[Z_{k}\right] . e_{J *} c_{d-|J|-k}\left(D_{J}\right) \prod_{j \in J}\left(\frac{-a_{j}}{a_{j}+1}\right)
$$

does not depend on the choice of the log-desingularization $\rho: Y \rightarrow X$. 
Corollary 2.2. Let $\rho^{*} c_{1}(X)=\left[-\rho^{*} K_{X}\right]$ be the pullback of the anticanonical class of $X$. Then the intersection number

$$
\sum_{\emptyset \subseteq J \subseteq I} \rho^{*} c_{1}(X)^{k} \cdot e_{J *} c_{d-|J|-k}\left(D_{J}\right) \prod_{j \in J}\left(\frac{-a_{j}}{a_{j}+1}\right)
$$

is independent of the log-desingularization $\rho$.

In the case $k=1$ (cf. Equation (4)), this theorem has been proved in Bat00, Corollary 3.9].

We observe that the intersection number $\left[Z_{1}\right] \ldots .\left[Z_{k}\right] . c_{d-k}^{s t r}(X)$ can be computed by a formula that does not involve stringy Chern classes of the singular variety $X$, but only usual Chern classes of smooth subvarieties $D_{J} \subseteq Y$. We give below a proof of Theorem 2.1, which does not use the general definiton of stringy Chern classes, but only the definition of the stringy Euler number (2) and its independence of logdesingularization.

Proof of Theorem 2.1. Let us denote the number

$$
\sum_{\emptyset \subseteq J \subseteq I} \rho^{*}\left[Z_{1}\right] \ldots . \rho^{*}\left[Z_{k}\right] . e_{J *} c_{d-|J|-k}\left(D_{J}\right) \prod_{j \in J}\left(\frac{-a_{j}}{a_{j}+1}\right)
$$

by $\imath_{\rho}\left(Z_{1}, \ldots, Z_{k}\right)$. It is clear that the map

$$
\imath_{\rho}: \operatorname{Pic}(X)_{\mathbb{Q}}^{k} \rightarrow \mathbb{Q},\left(\left[Z_{1}\right], \ldots,\left[Z_{k}\right]\right) \mapsto \imath_{\rho}\left(Z_{1}, \ldots, Z_{k}\right)
$$

is symmetric and multilinear. Since the group $\operatorname{Pic}(X)$ is generated by classes of very ample Cartier divisors, it is enough to show the statement of Theorem 2.1 in the case when $Z_{1}, \ldots, Z_{k}$ are very ample Cartier divisors. For any sequence of positive integers $n_{1}, \ldots, n_{k}$, the linear combination $n_{1}\left[Z_{1}\right]+\cdots+n_{k}\left[Z_{k}\right]$ represents a class of a very ample Cartier divisor $Z$ on $X$. It follows from the symmetry and multilinearity of $\iota_{\rho}$ that $\imath_{\rho}(Z, Z, \ldots, Z)$ is a homogeneous polynomial of degree $k$ in $n_{1}, \ldots, n_{k}$ whose coefficients are the rational numbers $\imath_{\rho}\left(Z_{i_{1}}, Z_{i_{2}}, \ldots, Z_{i_{k}}\right)$, where $1 \leq i_{1}, \ldots, i_{k} \leq k$. Therefore, it is enough to prove the statement of Theorem 2.1 only for the rational numbers $\imath_{\rho}(Z, Z, \ldots, Z)$, where $Z$ is a generic very ample Cartier divisor on $X$. By Theorem of Bertini, we can assume that $Z^{\prime}:=\rho^{-1}(Z)$ is a smooth divisor on $Y$ and the restriction of $\rho$ to $Z^{\prime}$ defines a log-desingularization of $Z$ with the exceptional divisors $D_{i}^{\prime}:=D_{i} \cap Z^{\prime}$ such that $K_{Z^{\prime}}=\rho^{*} K_{Z}+a_{1} D_{1}^{\prime}+\cdots+a_{s} D_{s}^{\prime}$. One can compute the stringy Euler number of $Z$ by

$$
e_{s t r}(Z)=\sum_{\emptyset \subseteq J \subseteq I} e\left(D_{J}^{\prime}\right) \prod_{j \in J}\left(\frac{-a_{j}}{a_{j}+1}\right),
$$

where $e\left(D_{J}^{\prime}\right)$ denotes the usual Euler number of the smooth variety $D_{J}^{\prime}=D_{J} \cap Z^{\prime}$ (cf. Equation (2) and [Bat98, Definition 3.3]). Now we apply Equation (12) to each 
smooth divisor $D_{J}^{\prime} \subseteq D_{J}$ and obtain

$$
e\left(D_{J}^{\prime}\right)=\sum_{k=1}^{d-|J|}(-1)^{k-1}\left[D_{J}^{\prime}\right]^{k} \cdot c_{d-|J|-k}\left(D_{J}\right)=\sum_{k=1}^{d-|J|}(-1)^{k-1} \rho^{*}[Z]^{k} \cdot e_{J *} c_{d-|J|-k}\left(D_{J}\right)
$$

because the projection formula for the proper morphism $e_{J}: D_{J} \hookrightarrow Y$ implies

$$
\begin{aligned}
\rho^{*}[Z]^{k} \cdot e_{J *} c_{d-|J|-k}\left(D_{J}\right) & =e_{J *}\left(e_{J}{ }^{*} \rho^{*}[Z]^{k} \cdot c_{d-|J|-k}\left(D_{J}\right)\right)=e_{J}^{*} \rho^{*}[Z]^{k} \cdot c_{d-|J|-k}\left(D_{J}\right) \\
& =e_{J}{ }^{*}\left[Z^{\prime}\right]^{k} \cdot c_{d-|J|-k}\left(D_{J}\right)=\left[D_{J}^{\prime}\right]^{k} \cdot c_{d-|J|-k}\left(D_{J}\right) .
\end{aligned}
$$

Therefore, the stringy Euler number $e_{s t r}(Z)$ of $Z$ has the form

$$
\begin{aligned}
e_{s t r}(Z) & =\sum_{\emptyset \subseteq J \subseteq I}\left(\sum_{k=1}^{d-|J|}(-1)^{k-1} \rho^{*}[Z]^{k} \cdot e_{J *} c_{d-|J|-k}\left(D_{J}\right)\right) \prod_{j \in J}\left(\frac{-a_{j}}{a_{j}+1}\right) \\
& =\sum_{k \geq 1}(-1)^{k-1} \rho^{*}[Z]^{k}\left(\sum_{\emptyset \subseteq J \subseteq I} e_{J *} c_{d-|J|-k}\left(D_{J}\right) \prod_{j \in J}\left(\frac{-a_{j}}{a_{j}+1}\right)\right) .
\end{aligned}
$$

For any positive integer $n$, the class $n[Z]$ can be again represented by a generic very ample Cartier divisor $Z^{(n)}$ such that $\rho^{-1}\left(Z^{(n)}\right)$ is smooth. So we can repeat the same arguments for $Z^{(n)}$ to obtain that the stringy Euler number

$$
e_{s t r}\left(Z^{(n)}\right)=\sum_{k \geq 1}(-1)^{k-1} n^{k} \rho^{*}[Z]^{k}\left(\sum_{\emptyset \subseteq J \subseteq I} e_{J *} c_{d-|J|-k}\left(D_{J}\right) \prod_{j \in J}\left(\frac{-a_{j}}{a_{j}+1}\right)\right)
$$

is a polynomial $P$ in $n$ whose $k$-th coefficient

$$
\rho^{*}[Z]^{k}\left(\sum_{\emptyset \subseteq J \subseteq I} e_{J *} c_{d-|J|-k}\left(D_{J}\right) \prod_{j \in J}\left(\frac{-a_{j}}{a_{j}+1}\right)\right)
$$

is equal to $\imath_{\rho}(Z, \ldots, Z)$. Since the stringy Euler number of $Z^{(n)}$ does not depend on the choice of the log-desingularization $\rho$ [Bat98, Theorem 3.4], the same is true for the polynomial $P$ and hence for its $k$-th coefficient $\imath_{\rho}(Z, \ldots, Z)$.

\section{Stringy Chern Classes on toric varieties}

It is well-known that singularities of an arbitrary $\mathbb{Q}$-Gorenstein toric variety $X$ are log-terminal. Moreover, the stringy Euler number $e_{s t r}(X)$ of the toric variety $X$ can be computed combinatorially using cones of maximal dimension in the associated fan $\Sigma$ [Bat98, Proposition 4.10] In this section, we give a combinatorial formula of all stringy Chern classes of arbitrary $\mathbb{Q}$-Gorenstein toric varieties using the intrinsic information provided by the associated fans. We apply this formula to compute intersection numbers $\left[D_{1}\right] \ldots \ldots\left[D_{k}\right] . c_{d-k}^{s t r}(X)$ via mixed volumes of certain polytopes.

We start with a well-know fact about Chern classes of smooth toric varieties:

Theorem 3.1. Let $V$ be a smooth toric variety associated with a fan $\Sigma$ in $N_{\mathbb{R}}$. Then the total Chern class of $V$ is

$$
c .(V)=\sum_{\sigma \in \Sigma}\left[V_{\sigma}\right]
$$


where $\left[V_{\sigma}\right]$ is the class of the closed torus orbit $V_{\sigma}$ corresponding to a cone $\sigma \in \Sigma$.

Theorem 3.2. Let $X$ be a $\mathbb{Q}$-Gorenstein toric variety associated with a fan $\Sigma$. Then the total stringy Chern class of $X$ is

$$
c_{\bullet}^{s t r}(X)=\sum_{\sigma \in \Sigma} v(\sigma) \cdot\left[X_{\sigma}\right],
$$

where $v(\sigma)=k ! \cdot \operatorname{vol}_{k}\left(\Theta_{\sigma}\right)$ and $\operatorname{vol}_{k}\left(\Theta_{\sigma}\right)$ is the $k$-dimensional volume of the lattice polytope $\Theta_{\sigma}$ obtained as the convex hull of the origin and the primitive lattice generators of all 1-dimensional faces of a cone $\sigma \in \Sigma(1)$.

Proof. Consider $\rho: Y \rightarrow X$ to be a log-desingularization of $X$ obtained by a refinement $\Sigma^{\prime}$ of the fan $\Sigma$. There is a natural bijection between the set of exceptional divisors $\left\{D_{1}, \ldots, D_{s}\right\}$ in $Y$ and the set of 1-dimensional cones $\Sigma^{\prime}(1) \backslash \Sigma(1)$. We denote by $\left\{D_{s+1}, \ldots, D_{r}\right\}$ the set of all remaining torus-invariant divisors in $Y$ whose elements one-to-one correspond to 1-dimensional cones in $\Sigma(1)$ and set $I:=\{1, \ldots, s\}$, $I^{\prime}:=I \cup\{s+1, \ldots, r\}$, and $a_{j}:=0$ for all $j \in I^{\prime} \backslash I$.

Let $\left\{u_{1}, \ldots, u_{r}\right\}=\left\{u_{i} \mid i \in I^{\prime}\right\}$ be the set of all primitive lattice generators of 1dimensional cones of $\Sigma^{\prime}(1)$ corresponding to all torus-invariant divisors $D_{1}, \ldots, D_{r}$. For any subset $J^{\prime} \subseteq I^{\prime}$, the subset $D_{J^{\prime}}=\cap_{j \in J^{\prime}} D_{j}$ is either empty or a smooth toric subvariety $Y_{\sigma^{\prime}}$ of $Y$. The latter holds if and only if the set $\left\{u_{j} \mid j \in J^{\prime}\right\}$ generates a cone $\sigma^{\prime} \in \Sigma^{\prime}$ of dimension $\left|J^{\prime}\right|$. Then the locally closed subset $D_{J^{\prime}}^{\circ}:=D_{J^{\prime}} \backslash\left(\cup_{i \in I^{\prime} \backslash J^{\prime}} D_{i}\right)$ is the dense open torus orbit $T_{\sigma^{\prime}}$ in $Y_{\sigma^{\prime}}$. By Theorem 3.1, we have $c .\left(T_{\sigma^{\prime}}\right)=\left[T_{\sigma^{\prime}}\right]$. This implies $c\left(\mathbb{1}_{D_{J^{\prime}}^{\circ}}\right)=\left[Y_{\sigma^{\prime}}\right]$. Using Equation (10), we get

$$
c_{\bullet}^{s t r}(X)=\rho_{*}\left(\sum_{\emptyset \subseteq J^{\prime} \subseteq I^{\prime}} c\left(\mathbb{1}_{D_{J^{\prime}}^{\circ}}\right) \prod_{j \in J^{\prime}}\left(\frac{1}{a_{j}+1}\right)\right)=\sum_{\sigma^{\prime} \in \Sigma^{\prime}} \rho_{*}\left[Y_{\sigma^{\prime}}\right] \prod_{u_{j} \in \sigma^{\prime}}\left(\frac{1}{a_{j}+1}\right) .
$$

Let us compute $\rho_{*}\left[Y_{\sigma^{\prime}}\right]$. If $\sigma \in \Sigma$ is the minimal cone of $\Sigma$ containing $\sigma^{\prime} \in \Sigma^{\prime}$, then $\rho\left(Y_{\sigma^{\prime}}\right)=X_{\sigma}$. In order to compute the corresponding cycle map $\rho_{*}: A_{\bullet}(Y) \rightarrow A$. $(X)$, we need to compare the dimensions of $Y_{\sigma^{\prime}}$ and $X_{\sigma}$. If $\operatorname{dim}\left(Y_{\sigma^{\prime}}\right)>\operatorname{dim}\left(X_{\sigma}\right)$, then $\rho_{*}\left[Y_{\sigma^{\prime}}\right]=0$. Otherwise, we have $\rho_{*}\left[Y_{\sigma^{\prime}}\right]=\left[X_{\sigma}\right]$.

Therefore, we get

$$
c_{\bullet}^{s t r}(X)=\sum_{\sigma \in \Sigma}\left(\sum_{\substack{\sigma^{\prime} \in \Sigma^{\prime}, \sigma^{\prime} \leq \sigma \\ \operatorname{dim}\left(\sigma^{\prime}\right)=\operatorname{dim}(\sigma)}} \prod_{u_{j} \in \sigma^{\prime}}\left(\frac{1}{a_{j}+1}\right)\right) \cdot\left[X_{\sigma}\right] .
$$

Furthermore, $\prod_{u_{j} \in \sigma^{\prime}}\left(\frac{1}{a_{j}+1}\right)=v\left(\sigma^{\prime}\right)$ for every cone $\sigma^{\prime} \in \Sigma^{\prime}$ and this implies

$$
c_{\bullet}^{s t r}(X)=\sum_{\sigma \in \Sigma}\left(\sum_{\substack{\sigma^{\prime} \in \Sigma^{\prime}, \sigma^{\prime} \leq \sigma \\ \operatorname{dim}\left(\sigma^{\prime}\right)=\operatorname{dim}(\sigma)}} v\left(\sigma^{\prime}\right)\right) \cdot\left[X_{\sigma}\right]=\sum_{\sigma \in \Sigma} v(\sigma) \cdot\left[X_{\sigma}\right] .
$$


Corollary 3.3. Let $X$ be a d-dimensional $\mathbb{Q}$-Gorenstein toric variety associated with a fan $\Sigma$. Then the $k$-th stringy Chern class of $X(0 \leq k \leq d)$ is

$$
c_{k}^{s t r}(X)=\sum_{\sigma \in \Sigma(k)} v(\sigma) \cdot\left[X_{\sigma}\right] .
$$

The above formula allows to compute combinatorially the intersection number of the stringy Chern class $c_{d-1}^{s t r}(X)$ of a $d$-dimensional projective $\mathbb{Q}$-Gorenstein toric variety $X$ corresponding to a fan $\Sigma$ with an arbitrary torus-invariant $\mathbb{Q}$-Cartier divisor $D=\sum_{\rho \in \Sigma(1)} a_{\rho} D_{\rho}$ on $X$.

Therefore, we define for any $(d-1)$-dimensional cone $\sigma \in \Sigma(d-1)$ the rational number $l_{D}(\sigma)$ : Consider two $d$-dimensional cones $\sigma^{\prime}, \sigma^{\prime \prime} \in \Sigma(d)$ such that $\sigma=\sigma^{\prime} \cap \sigma^{\prime \prime}$. Denote by $m_{\sigma^{\prime}}$ and $m_{\sigma^{\prime \prime}}$ elements in $M_{\mathbb{Q}}$ that are defined by the conditions $\left\langle m_{\sigma^{\prime}}, u_{\rho}\right\rangle=$ $-a_{\rho} \forall \rho \subseteq \sigma^{\prime}$ respectively $\left\langle m_{\sigma^{\prime \prime}}, u_{\rho}\right\rangle=-a_{\rho} \forall \rho \subseteq \sigma^{\prime \prime}$, where $\rho \in \Sigma(1)$ and $u_{\rho} \in N$ denotes its primitive lattice generator. Now choose the primitive lattice generator $u$ of the 1-dimensional sublattice $M(\sigma):=\left\{m \in M \mid\left\langle m, u^{\prime}\right\rangle=0 \forall u^{\prime} \in \sigma\right\}$ such that $\left.u\right|_{\sigma^{\prime}} \leq 0$ and $\left.u\right|_{\sigma^{\prime \prime}} \geq 0$. Since $m_{\sigma^{\prime}}-m_{\sigma^{\prime \prime}}$ vanishes on $\sigma$, there exists a unique number $l_{D}(\sigma) \in \mathbb{Q}$ such that $m_{\sigma^{\prime}}-m_{\sigma^{\prime \prime}}=l_{D}(\sigma) \cdot u$.

Proposition 3.4. Let $X$ be a d-dimensional projective $\mathbb{Q}$-Gorenstein toric variety associated with a fan $\Sigma$ and $D$ a torus-invariant $\mathbb{Q}$-Cartier divisor on $X$. Then

$$
[D] . c_{d-1}^{s t r}(X)=\sum_{\sigma \in \Sigma(d-1)} v(\sigma) \cdot l_{D}(\sigma),
$$

where the rational number $l_{D}(\sigma) \in \mathbb{Q}$ is defined as above.

Proof. Using Corollary 3.3, we obtain

$$
[D] . c_{d-1}^{s t r}(X)=\sum_{\sigma \in \Sigma(d-1)} v(\sigma) \cdot[D] \cdot\left[X_{\sigma}\right] .
$$

It remains to apply the equality $[D] \cdot\left[X_{\sigma}\right]=l_{D}(\sigma)$ (cf. CLS11, Proposition 6.3.8]) for every cone $\sigma \in \Sigma(d-1)$.

Now we compute intersection numbers $[D]^{k} \cdot c_{d-k}^{s t r}(X)$, where $D=\sum_{\rho \in \Sigma(1)} a_{\rho} D_{\rho}$ is a semiample torus-invariant $\mathbb{Q}$-Cartier divisor on the toric variety $X$. Consider the corresponding convex rational polytope $\Delta_{D}$ of dimension $\leq d$ defined as

$$
\Delta_{D}:=\left\{y \in M_{\mathbb{R}} \mid\left\langle y, u_{\rho}\right\rangle \geq-a_{\rho} \forall \rho \in \Sigma(1)\right\} \subseteq M_{\mathbb{R}},
$$

where $u_{\rho} \in N$ is the primitiv lattice generator of a 1-dimensional cone $\rho \in \Sigma(1)$. Let $\sigma \in \Sigma(d-k)$ be a $(d-k)$-dimensional cone of the fan $\Sigma$. Denote by $\Delta_{D}^{\sigma}$ a face of $\Delta_{D}$ of dimension $\leq k$ defined as

$$
\Delta_{D}^{\sigma}:=\left\{y \in \Delta_{D} \mid\left\langle y, u_{\rho}\right\rangle=-a_{\rho} \forall \rho \in \Sigma(1) \text { with } \rho \in \sigma\right\} \subseteq M_{\mathbb{R}} .
$$

The volume of the rational polytope $\Delta_{D}^{\sigma}$ is defined as

$$
v\left(\Delta_{D}^{\sigma}\right):=k ! \cdot \operatorname{vol}_{k}\left(\Delta_{D}^{\sigma}\right) \in \mathbb{Q},
$$


where $\operatorname{vol}_{k}\left(\Delta_{D}^{\sigma}\right)$ is the volume of $\Delta_{D}^{\sigma}$ with respect to the $k$-dimensional sublattice $M(\sigma)=\left\{m \in M \mid\left\langle m, u^{\prime}\right\rangle=0 \forall u^{\prime} \in \sigma\right\}$ of $M$. In particular, one has $v\left(\Delta_{D}^{\sigma}\right)=0$ if $\operatorname{dim}\left(\Delta_{D}^{\sigma}\right)<k$.

Theorem 3.5. Let $X$ be a d-dimensional projective $\mathbb{Q}$-Gorenstein toric variety associated with a fan $\Sigma$ and $D$ a semiample torus-invariant $\mathbb{Q}$-Cartier divisor on $X$. Then

$$
[D]^{k} \cdot c_{d-k}^{s t r}(X)=\sum_{\sigma \in \Sigma(d-k)} v(\sigma) \cdot v\left(\Delta_{D}^{\sigma}\right)
$$

where the face $\Delta_{D}^{\sigma}$ of $\Delta_{D}$ and $v\left(\Delta_{D}^{\sigma}\right)$ are defined as above $(0 \leq k \leq d)$.

Proof. By Corollary 3.3, we have

$$
[D]^{k} \cdot c_{d-k}^{s t r}(X)=\sum_{\sigma \in \Sigma(d-k)} v(\sigma) \cdot[D]^{k} \cdot\left[X_{\sigma}\right] .
$$

Let $D^{\sigma}$ be the restriction of the semiample torus-invariant $\mathbb{Q}$-Cartier divisor $D$ to the $k$-dimensional toric subvariety $X_{\sigma}$ of $X$. Then $[D]^{k} \cdot\left[X_{\sigma}\right]$ is the intersection number $\left[D^{\sigma}\right]^{k}$ of the semiample torus-invariant $\mathbb{Q}$-Cartier divisor $D^{\sigma}$ on the $k$-dimensional variety $X_{\sigma}$. It remains to note that the number $\left[D^{\sigma}\right]^{k}$ equals $v\left(\Delta_{D}^{\sigma}\right)$ (cf. CLS11, Section 13.4]).

Using Theorem 3.5 and Corollary 1.4 respectively 1.7 , we derive combinatorial formulas for the stringy Euler number of generic hypersurfaces and complete intersections in toric varieties:

Corollary 3.6. Let $X$ be a d-dimensional projective $\mathbb{Q}$-Gorenstein toric variety associated with a fan $\Sigma$ and $D$ a semiample torus-invariant Cartier divisor on $X$ together with the corresponding lattice polytope $\Delta_{D}$. Denote by $Z \subseteq X$ a generic semiample Cartier divisor such that $[Z]=[D]$. Then the stringy Euler number of $Z$ is

$$
e_{s t r}(Z)=c_{d-1}^{s t r}(Z)=\sum_{k=0}^{d-1}(-1)^{k} \sum_{\sigma \in \Sigma(d-1-k)} v(\sigma) \cdot v\left(\Delta_{D}^{\sigma}\right)
$$

where $\Delta_{D}^{\sigma}$ is a face of $\Delta_{D}$ corresponding to a cone $\sigma \in \Sigma$. If $[Z]=c_{1}(X)$, the formula simplifies to

$$
e_{s t r}(Z)=c_{d-1}^{s t r}(Z)=\sum_{k=0}^{d-3}(-1)^{k} \sum_{\sigma \in \Sigma(d-1-k)} v(\sigma) \cdot v\left(\Delta_{D}^{\sigma}\right) .
$$

Corollary 3.7. Let $X$ be a d-dimensional projective $\mathbb{Q}$-Gorenstein toric variety associated with a fan $\Sigma$ and $D$ a semiample torus-invariant Cartier divisor on $X$ together with the corresponding lattice polytope $\Delta_{D}$. Denote by $Z_{1}, \ldots, Z_{r} \subseteq X$ generic semiample Cartier divisors such that $\left[Z_{1}\right]=\ldots=\left[Z_{r}\right]=[D]$. Then the stringy Euler 
number of the complete intersection $Z_{1} \cap \ldots \cap Z_{r}$ is

$$
c_{d-r}^{s t r}\left(Z_{1} \cap \ldots \cap Z_{r}\right)=\sum_{k=0}^{d-r}(-1)^{k}\left(\begin{array}{c}
k+r-1 \\
r-1
\end{array}\right) \sum_{\sigma \in \Sigma(d-r-k)} v(\sigma) \cdot v\left(\Delta_{D}^{\sigma}\right),
$$

where $\Delta_{D}^{\sigma}$ is a face of $\Delta_{D}$ as above.

One can generalize Theorem 3.5 and combinatorially compute intersection numbers $\left[D_{1}\right] . \ldots \cdot\left[D_{k}\right] \cdot c_{d-k}^{s t r}(X)$, where $D_{1}, \ldots, D_{k}$ are different semiample torus-invariant $\mathbb{Q}$-Cartier divisors on $X$. For this purpose, we use mixed volumes of faces of some convex rational polytopes.

Theorem 3.8. Let $X$ be a d-dimensional projective $\mathbb{Q}$-Gorenstein toric variety associated with a fan $\Sigma$ and $D_{1}, \ldots, D_{k}$ semiample torus-invariant $\mathbb{Q}$-Cartier divisors on $X$. Then

$$
\left[D_{1}\right] \ldots \cdot\left[D_{k}\right] \cdot c_{d-k}^{s t r}(X)=\sum_{\sigma \in \Sigma(d-k)} v(\sigma) \cdot v\left(\Delta_{D_{1}}^{\sigma}, \ldots, \Delta_{D_{k}}^{\sigma}\right),
$$

where $\Delta_{D_{i}}^{\sigma}$ is a face of $\Delta_{D_{i}}(1 \leq i \leq k)$ corresponding to a cone $\sigma \in \Sigma$ and $v\left(\Delta_{D_{1}}^{\sigma}, \ldots, \Delta_{D_{k}}^{\sigma}\right)$ denotes the mixed volume of the polytopes $\Delta_{D_{1}}^{\sigma}, \ldots, \Delta_{D_{k}}^{\sigma}$ with respect to the sublattice $M(\sigma) \subseteq M$.

Proof. Let $\sigma \in \Sigma(d-k)$ be a $(d-k)$-dimensional cone. Then we restrict the semiample torus-invariant $\mathbb{Q}$-Cartier divisors $D_{1}, \ldots, D_{k}$ to the corresponding projective $k$-dimensional toric subvariety $X_{\sigma}$ of $X$ and obtain $k$ semiample torus-invariant $\mathbb{Q}$ Cartier divisors $D_{1}^{\sigma}, \ldots, D_{k}^{\sigma}$ on $X_{\sigma}$. It remains to apply Corollary 3.3 and the formula in [Ful93, Section 5.4] that claims that the intersection number

$$
\left[D_{1}\right] \ldots \ldots\left[D_{k}\right] \cdot\left[X_{\sigma}\right]=\left[D_{1}^{\sigma}\right] \ldots \ldots \cdot\left[D_{k}^{\sigma}\right]
$$

can be computed as the mixed volume $v\left(\Delta_{D_{1}}^{\sigma}, \ldots, \Delta_{D_{k}}^{\sigma}\right)$ of the polytopes $\Delta_{D_{i}}^{\sigma}$.

\section{Stringy LibGober-Wood IDEntity FOR TORIC VARIETIES}

The identity

$$
\left.\frac{d^{2}}{d u^{2}} E(V ; u, 1)\right|_{u=1}=\frac{3 d^{2}-5 d}{12} c_{d}(V)+\frac{1}{6} c_{1}(V) \cdot c_{d-1}(V)
$$

has been proved by Libgober and Wood [LW90] for arbitrary smooth $d$-dimensional projective varieties $V$. This identity is equivalent to

$$
\sum_{0 \leq p, q \leq d}(-1)^{p+q} h^{p, q}(V)\left(p-\frac{d}{2}\right)^{2}=\frac{d}{12} c_{d}(V)+\frac{1}{6} c_{1}(V) \cdot c_{d-1}(V)
$$

and so the intersection number $c_{1}(V) \cdot c_{d-1}(V)$ can be expressed via the Hodge numbers $h^{p, q}(V)$ of $V$ [Bor97].

There exists a stringy version of the Libgober-Wood identity

$$
\left.\frac{d^{2}}{d u^{2}} E_{s t r}(X ; u, 1)\right|_{u=1}=\frac{3 d^{2}-5 d}{12} c_{d}^{s t r}(X)+\frac{1}{6} c_{1}(X) \cdot c_{d-1}^{s t r}(X),
$$


which holds for any $d$-dimensional projective variety $X$ with at worst log-terminal singularities [Bat00, Theorem 3.8] (cf. Equation (3)).

Moreover, if the singularities of $X$ are at worst canonical Gorenstein and the stringy $E$-function $E_{s t r}(X ; u, v)$ is a polynomial $\sum_{p, q} \psi_{p, q} u^{p} v^{q}$, then one can define the stringy Hodge numbers $h_{s t r}^{p, q}(X)$ of $X$ [Bat98, Bat00] as

$$
h_{s t r}^{p, q}(X):=(-1)^{p+q} \psi_{p, q} .
$$

In this case, the stringy Libgober-Wood identity can be equivalently reformulated [Bat00, Corollary 3.10] as

$$
\sum_{0 \leq p, q \leq d}(-1)^{p+q} h_{s t r}^{p, q}(X)\left(p-\frac{d}{2}\right)^{2}=\frac{d}{12} c_{d}^{s t r}(X)+\frac{1}{6} c_{1}(X) \cdot c_{d-1}^{s t r}(X) .
$$

In this section, we are interested in a combinatorial interpretation of these stringy Libgober-Wood identities (13), (14) for arbitrary $d$-dimensional projective $\mathbb{Q}$-Gorenstein toric varieties $X$ associated with a fan $\Sigma$. Let $q_{X}$ be the smallest positive integer such that $q_{X} K_{X}$ is a Cartier divisor. The number $q_{X}$ is called Gorenstein index of $X$. Note that the stringy $E$-function $E_{s t r}(X ; u, v)$ of such a toric variety $X$ can be computed combinatorially as

$$
E_{s t r}(X ; u, v)=(u v-1)^{d} \sum_{\sigma \in \Sigma} \sum_{n \in \sigma^{\circ} \cap N}(u v)^{\kappa(n)},
$$

where $\kappa$ is the $\Sigma$-piecewise linear function corresponding to the anticanonical divisor of $X$ and $\sigma^{\circ}$ is the relative interior of a cone $\sigma \in \Sigma$ [Bat98, Theorem 4.3]. We remark that $\kappa$ has value -1 on every primitive lattice generator of a 1-dimensional cone $\sigma \in \Sigma(1)$ and that the value $\kappa(n)(n \in N)$ belongs to $\frac{1}{q_{X}} \mathbb{Z}$.

First, we show that the stringy $E$-function $E_{s t r}(X ; u, v)$ is a polynomial with nonnegative integral coefficients $\psi_{\alpha}(\Sigma)$ in nonnegative rational powers $\alpha \in[0, d] \cap \frac{1}{q_{X}} \mathbb{Z}$ of $u v$ :

Proposition 4.1. Let $X$ be a d-dimensional projective $\mathbb{Q}$-Gorenstein toric variety of Gorenstein index $q_{X}$ associated with a fan $\Sigma$ in $N_{\mathbb{R}}$ and $\Sigma^{\prime}$ a simplicial subdivision of the fan $\Sigma$ such that $\Sigma^{\prime}(1)=\Sigma(1)$. For any cone $\sigma \in \Sigma^{\prime}$, we denote by $\square_{\sigma}^{\circ}$ the relative interior of the parallelepiped $\square_{\sigma}$ spanned by the primitive lattice generators of the cone $\sigma$. Then the stringy E-function can be computed as a finite sum

$$
E_{s t r}(X ; u, v)=\sum_{\sigma \in \Sigma^{\prime}}(u v-1)^{d-\operatorname{dim}(\sigma)} \sum_{n^{\prime} \in \square_{\sigma}^{\circ} \cap N}(u v)^{\operatorname{dim}(\sigma)+\kappa\left(n^{\prime}\right)} .
$$

Moreover, the stringy E-function can be written as a finite sum

$$
E_{s t r}(X ; u, v)=\sum_{\alpha \in[0, d] \cap \frac{1}{q_{X}} \mathbb{Z}} \psi_{\alpha}(\Sigma)(u v)^{\alpha},
$$

where the coefficients $\psi_{\alpha}(\Sigma)$ are nonnegative integers satisfying the conditions $\psi_{0}(\Sigma)=$ $\psi_{d}(\Sigma)=1$ and $\psi_{\alpha}(\Sigma)=\psi_{d-\alpha}(\Sigma)$ for all $\alpha \in[0, d] \cap \frac{1}{q_{X}} \mathbb{Z}$. 
Proof. Any $s$-dimensional simplicial cone $\sigma$ of $\Sigma^{\prime}(s)$ is generated by $s$ linearly independent primitive lattice vectors $u_{1}, \ldots, u_{s}$. Therefore, any lattice point $n \in \sigma^{\circ} \cap N$ has a unique representation as a sum $n=n^{\prime}+n^{\prime \prime}$, where $n^{\prime}=\sum_{i=1}^{s} \lambda_{i} u_{i} \in \square_{\sigma}^{\circ} \cap N$ $\left(0 \leq \lambda_{i} \leq 1\right)$ and $n^{\prime \prime}$ is a linear combination $n^{\prime \prime}=\sum_{i=1}^{s} k_{i} u_{i}$ with nonnegative integral coefficients $k_{i}$. Therefore, one has

$$
\begin{aligned}
(u v-1)^{s} \sum_{n \in \sigma^{\circ} \cap N}(u v)^{\kappa(n)} & =(u v-1)^{s} \sum_{n^{\prime} \in \square_{\sigma}^{\circ} \cap N}(u v)^{\kappa\left(n^{\prime}\right)} \prod_{i=1}^{s}\left(\sum_{k_{i} \in \mathbb{Z}_{\geq 0}}(u v)^{-k_{i}}\right) \\
& =(u v-1)^{s} \sum_{n^{\prime} \in \square_{\sigma}^{\circ} \cap N}(u v)^{\kappa\left(n^{\prime}\right)} \cdot\left(\frac{1}{1-(u v)^{-1}}\right)^{s}=\sum_{n^{\prime} \in \square_{\sigma}^{\circ} \cap N}(u v)^{s+\kappa\left(n^{\prime}\right)}
\end{aligned}
$$

and the first statement of Proposition 4.1 follows from Equation (15). Since $\kappa$ has value -1 on every primitive lattice generator $u_{i}$ and $q_{X} \cdot \kappa(n) \in \mathbb{Z}$ for all $n \in N$, we obtain that $s+\kappa\left(n^{\prime}\right)=s-\sum_{i=1}^{s} \lambda_{i}$ is a nonnegative rational number in $\frac{1}{q_{X}} \mathbb{Z}_{\geq 0}$. Therefore, $E_{s t r}(X ; u, v)$ can be written as a finite sum $E_{s t r}(X ; u, v)=\sum_{\alpha} \psi_{\alpha}(\Sigma)(u v)^{\alpha}$ for some integral coefficients $\psi_{\alpha}(\Sigma)$ and some nonnegative rational numbers $\alpha$ in $\frac{1}{q_{X}} \mathbb{Z}_{\geq 0}$. The Poincaré duality [Bat98, Theorem 3.7] for the stringy E-function

$$
E_{s t r}(X ; u, v)=(u v)^{d} E_{s t r}\left(X ; u^{-1}, v^{-1}\right)
$$

delivers the equalities $\psi_{\alpha}(\Sigma)=\psi_{d-\alpha}(\Sigma)$. This implies $\alpha \leq d$ as soon as $\psi_{\alpha}(\Sigma) \neq 0$. Therefore, we obtain

$$
E_{s t r}(X ; u, v)=\sum_{\alpha \in[0, d] \cap \frac{1}{q_{X}} \mathbb{Z}} \psi_{\alpha}(\Sigma)(u v)^{\alpha} .
$$

The nonnegativity of the coefficients $\psi_{\alpha}(\Sigma)$ can be shown using an interpretation of the coefficients $\psi_{\alpha}(\Sigma)$ as dimensions of graded homogenous components of a graded artinian ring $R$ obtained as a quotient of a graded Cohen-Macaulay ring $S$ by a regular sequence of homogeneous elements (cf. [Bat93, Theorem 2.11]).

Corollary 4.2. Let $X$ be a 2 -dimensional projective $\mathbb{Q}$-Gorenstein toric variety associated with a fan $\Sigma$ in $N_{\mathbb{R}}$. Then

$$
E_{s t r}(X ; u, v)=(u v-1)^{2}+\sum_{\substack{n \in N \\ \kappa(n)=-1}} u v+\sum_{\substack{n \in N \\-1<\kappa(n)<0}}\left((u v)^{2+\kappa(n)}+(u v)^{-\kappa(n)}\right) .
$$

Proof. We do not need a subdivision $\Sigma^{\prime}$ of the fan $\Sigma$ because every cone $\sigma \in \Sigma$ already is simplicial. Therefore, we set $\Sigma^{\prime}=\Sigma$. By Proposition 4.1, we obtain

$$
\begin{aligned}
E_{s t r}(X ; u, v) & =\sum_{\sigma \in \Sigma}(u v-1)^{2-\operatorname{dim}(\sigma)} \sum_{n \in \square_{\sigma}^{\circ} \cap N}(u v)^{\operatorname{dim}(\sigma)+\kappa(n)} \\
& =(u v-1)^{2}+\sum_{\sigma \in \Sigma(1)}(u v-1)+\sum_{\sigma \in \Sigma(2)} \sum_{n \in \square_{\sigma}^{\circ} \cap N}(u v)^{2+\kappa(n)} .
\end{aligned}
$$

For any 2-dimensional cone $\sigma \in \Sigma(2)$, the set $\left\{x \in N_{\mathbb{R}}: \kappa(x)=-1\right\}$ divides the parallelogram $\square_{\sigma}$ into two isomorphic lattice triangles $\triangle_{\leq-1}^{\sigma}$ and $\triangle_{\geq-1}^{\sigma}$. Let $u_{1}, u_{2}$ be the primitive lattice generators of $\sigma$. We can write every lattice point $n \in \square_{\sigma}$ 
as a linear combination $n=\lambda_{1} u_{1}+\lambda_{2} u_{2}$ with rational coefficients $\lambda_{1}, \lambda_{2} \in[0,1]$. A lattice point $n \in \square_{\sigma}$ belongs to the triangle $\triangle_{\geq-1}^{\sigma}$ if and only if the lattice point $n^{*}:=u_{1}+u_{2}-n$ belongs to the triangle $\triangle_{\leq-1}^{\sigma}$. Since the boundary of the lattice parallelogram $\square_{\sigma}$ has no lattice points except vertices, we can use the bijection $n \leftrightarrow n^{*}$ together with the equation $\kappa(n)+\kappa\left(n^{*}\right)=-2$ to obtain

$$
\sum_{n \in \square_{\sigma}^{\circ} \cap N}(u v)^{2+\kappa(n)}=1+\sum_{\substack{n \in \sigma^{\circ} \cap N \\ \kappa(n)=-1}} u v+\sum_{\substack{n \in \sigma \cap N \\-1<\kappa(n)<0}}\left((u v)^{2+\kappa(n)}+(u v)^{-\kappa(n)}\right) .
$$

It remains to apply the equalities $|\Sigma(1)|=|\Sigma(2)|$ and

$$
\sum_{\sigma \in \Sigma(1)} u v+\sum_{\sigma \in \Sigma(2)} \sum_{\substack{n \in \sigma^{\circ} \cap N \\ \kappa(n)=-1}} u v=\sum_{\substack{n \in N \\ \kappa(n)=-1}} u v .
$$

The equality $E_{s t r}(X ; u, v)=\sum_{\alpha} \psi_{\alpha}(\Sigma)(u v)^{\alpha}$ in Proposition 4.1 suggests that the nonnegative integral coefficients $\psi_{\alpha}(\Sigma)$ may be interpreted as generalized stringy Hodge numbers $h_{s t r}^{\alpha, \alpha}(X)$ of the toric variety $X$ for some rational numbers $\alpha \in[0, d] \cap$ $\frac{1}{q_{X}} \mathbb{Z}$

The following theorem presents a combinatorial interpretation for the second version of the stringy Libgober-Wood identity (14) using the generalized stringy Hodge numbers of the toric variety $X$.

Theorem 4.3. Let $X$ be a d-dimensional projective $\mathbb{Q}$-Gorenstein toric variety of Gorenstein index $q_{X}$ associated with a fan $\Sigma$ and $-K_{X}=\sum_{\rho \in \Sigma(1)} D_{\rho}$ the anticanonical torus-invariant $\mathbb{Q}$-Cartier divisor on $X$. Then the stringy Libgober-Wood identity is equivalent to

$$
\sum_{\alpha \in[0, d] \cap \frac{1}{q_{X}} \mathbb{Z}} \psi_{\alpha}(\Sigma)\left(\alpha-\frac{d}{2}\right)^{2}=\frac{d}{12} v(\Sigma)+\frac{1}{6} \sum_{\sigma \in \Sigma(d-1)} v(\sigma) \cdot l_{-K_{X}}(\sigma),
$$

where $\psi_{\alpha}(\Sigma)$ are nonnegative integers as above, $v(\Sigma):=\sum_{\sigma \in \Sigma(d)} v(\sigma)$, and $l_{-K_{X}}(\sigma) \in$ $\mathbb{Q}$ is the intersection number $\left[-K_{X}\right] .\left[X_{\sigma}\right]$ (cf. Proposition 3.4). If $-K_{X}$ is semiample, then

$$
\sum_{\alpha \in[0, d] \cap \frac{1}{q_{X}} \mathbb{Z}} \psi_{\alpha}(\Sigma)\left(\alpha-\frac{d}{2}\right)^{2}=\frac{d}{12} v(\Sigma)+\frac{1}{6} \sum_{\sigma \in \Sigma(d-1)} v(\sigma) \cdot v\left(\Delta_{-K_{X}}^{\sigma}\right),
$$

where $\Delta_{-K_{X}}^{\sigma}$ is a face of the rational polytope $\Delta_{-K_{X}}$ corresponding to a cone $\sigma \in \Sigma$ (cf. Theorem 3.5).

Proof. Using the equality $E_{s t r}(X ; u, v)=\sum_{\alpha} \psi_{\alpha}(\Sigma)(u v)^{\alpha}$ from Proposition 4.1, we obtain

$$
\left.\frac{d^{2}}{d u^{2}} E_{s t r}(X ; u, 1)\right|_{u=1}=\sum_{\alpha} \alpha \cdot(\alpha-1) \psi_{\alpha}(\Sigma)
$$


i.e., the stringy Libgober-Wood identity (13) is given as

$$
\sum_{\alpha}\left(\alpha^{2}-\alpha\right) \psi_{\alpha}(\Sigma)=\frac{3 d^{2}-5 d}{12} c_{d}^{s t r}(X)+\frac{1}{6} c_{1}(X) \cdot c_{d-1}^{s t r}(X) .
$$

Applying $\sum_{\alpha} \alpha \psi_{\alpha}(\Sigma)=\left.\frac{d}{d u} E_{s t r}(X ; u, 1)\right|_{u=1}=\frac{d}{2} c_{d}^{s t r}(X)$ [Bat00, Proposition 3.4] a short calculation yields

$$
\sum_{\alpha} \alpha^{2} \psi_{\alpha}(\Sigma)=\left(\frac{d}{12}+\frac{d^{2}}{4}\right) c_{d}^{s t r}(X)+\frac{1}{6} c_{1}(X) \cdot c_{d-1}^{s t r}(X)
$$

and implies

$$
\begin{aligned}
\sum_{\alpha} \psi_{\alpha}(\Sigma)\left(\alpha-\frac{d}{2}\right)^{2} & =\sum_{\alpha} \alpha^{2} \psi_{\alpha}(\Sigma)-d \sum_{\alpha} \alpha \psi_{\alpha}(\Sigma)+\frac{d^{2}}{4} \sum_{\alpha} \psi_{\alpha}(\Sigma) \\
& =\frac{d}{12} c_{d}^{s t r}(X)+\frac{1}{6} c_{1}(X) \cdot c_{d-1}^{s t r}(X)
\end{aligned}
$$

because $\sum_{\alpha} \psi_{\alpha}(\Sigma)=\left.E_{\text {str }}(X ; u, 1)\right|_{u=1}=c_{d}^{\text {str }}(X)$ [Bat00, Definition 2.1]. To finish, it remains to note that $c_{d}^{s t r}(X)=v(\Sigma)$ by Corollary 3.3 (cf. Bat98, Proposition 4.10]),

$$
c_{1}(X) \cdot c_{d-1}^{s t r}(X)=\left[-K_{X}\right] \cdot c_{d-1}^{s t r}(X)=\sum_{\sigma \in \Sigma(d-1)} v(\sigma) \cdot l_{-K_{X}}(\sigma)
$$

by Proposition 3.4, and

$$
c_{1}(X) \cdot c_{d-1}^{s t r}(X)=\left[-K_{X}\right] . c_{d-1}^{s t r}(X)=\sum_{\sigma \in \Sigma(d-1)} v(\sigma) \cdot v\left(\Delta_{-K_{X}}^{\sigma}\right)
$$

by Theorem 3.5 if $-K_{X}$ is semiample.

We formulate one more combinatorial version of the stringy Libgober-Wood identity containing only intrinsic informations coming from the associated fan $\Sigma$ of the toric variety $X$. To achieve this, we describe the left side of the stringy LibgoberWood identity (13) in pure combinatorial terms using Proposition 4.1.

Theorem 4.4. Let $X$ be a d-dimensional projective $\mathbb{Q}$-Gorenstein toric variety associated with a fan $\Sigma$ in $N_{\mathbb{R}}$ and $-K_{X}=\sum_{\rho \in \Sigma(1)} D_{\rho}$ the anticanonical torus-invariant $\mathbb{Q}$-Cartier divisor on $X$. Then the stringy Libgober-Wood identity is equivalent to

$$
\begin{aligned}
& 2 \cdot \sum_{\sigma \in \Sigma^{\prime}(d-2)}\left|\square_{\sigma}^{\circ} \cap N\right|+2 \cdot \sum_{\sigma \in \Sigma^{\prime}(d-1)} \sum_{n^{\prime} \in \square_{\sigma}^{\circ} \cap N}\left(d+\kappa\left(n^{\prime}\right)-1\right) \\
& \quad+\sum_{\sigma \in \Sigma^{\prime}(d)} \sum_{n^{\prime} \in \square_{\sigma}^{\circ} \cap N}\left(d+\kappa\left(n^{\prime}\right)\right)\left(d+\kappa\left(n^{\prime}\right)-1\right)=\frac{\left(3 d^{2}-5 d\right)}{12} v(\Sigma)+\frac{1}{6} c_{1}(X) \cdot c_{d-1}^{s t r}(X),
\end{aligned}
$$

where $\Sigma^{\prime}$ is a simplicial subdivision of the fan $\Sigma$ such that $\Sigma^{\prime}(1)=\Sigma(1), \square_{\sigma}^{\circ}$ is the relative interior of the parallelepiped $\square_{\sigma}$ spanned by the primitive lattice generators 
of a cone $\sigma \in \Sigma, \kappa$ is the $\Sigma$-piecewise linear function corresponding to $-K_{X}$, and $v(\Sigma)=\sum_{\sigma \in \Sigma(d)} v(\sigma)$. The rational number $c_{1}(X) . c_{d-1}^{s t r}(X) \in \mathbb{Q}$ is computable as

$$
c_{1}(X) \cdot c_{d-1}^{s t r}(X)=\sum_{\sigma \in \Sigma(d-1)} v(\sigma) \cdot l_{-K_{X}}(\sigma)
$$

where $l_{-K_{X}}$ is the intersection number $\left[-K_{X}\right] \cdot\left[X_{\sigma}\right]$ (cf. Proposition 3.4). If $-K_{X}$ is semiample, then

$$
c_{1}(X) \cdot c_{d-1}^{s t r}(X)=\sum_{\sigma \in \Sigma(d-1)} v(\sigma) \cdot v\left(\Delta_{-K_{X}}^{\sigma}\right)
$$

where $\Delta_{-K_{X}}^{\sigma}$ is a face of the rational polytope $\Delta_{-K_{X}}$ (cf. Theorem 3.5).

Proof. We derivate one summand of the stringy $E$-function

$$
E_{s t r}(X ; u, v)=\sum_{\sigma \in \Sigma^{\prime}}(u v-1)^{d-\operatorname{dim}(\sigma)} \sum_{n^{\prime} \in \square_{\sigma}^{\circ} \cap N}(u v)^{\operatorname{dim}(\sigma)+\kappa\left(n^{\prime}\right)} .
$$

from Proposition 4.1 inserted $v=1$ twice and get

$$
\begin{array}{r}
\frac{d^{2}}{d u^{2}}(u-1)^{d-s} \sum_{n^{\prime} \in \square_{\sigma}^{\circ} \cap N} u^{s+\kappa\left(n^{\prime}\right)}=(d-s)(d-1-s)(u-1)^{d-2-s} \sum_{n^{\prime} \in \square_{\sigma}^{\circ} \cap N} u^{s+\kappa\left(n^{\prime}\right)} \\
+2 \cdot(d-s)(u-1)^{d-1-s} \sum_{n^{\prime} \in \square_{\sigma}^{\circ} \cap N}\left(s+\kappa\left(n^{\prime}\right)\right) u^{s+\kappa\left(n^{\prime}\right)-1} \\
+(u-1)^{d-s} \sum_{n^{\prime} \in \square_{\sigma}^{\circ} \cap N}\left(s+\kappa\left(n^{\prime}\right)\right)\left(s+\kappa\left(n^{\prime}\right)-1\right) u^{s+\kappa\left(n^{\prime}\right)-2}
\end{array}
$$

where $\sigma$ is any $s$-dimensional cone of $\Sigma^{\prime}(s)$. Inserting $u=1$ the relevant cones of $\Sigma^{\prime}$ are these of dimension $d, d-1$, and $d-2$, i.e.,

$$
\begin{aligned}
\left.\frac{d^{2}}{d u^{2}} E_{s t r}(X ; u, 1)\right|_{u=1} & =2 \cdot \sum_{\sigma \in \Sigma^{\prime}(d-2)}\left|\square_{\sigma}^{\circ} \cap N\right|+2 \cdot \sum_{\sigma \in \Sigma^{\prime}(d-1)} \sum_{n^{\prime} \in \square_{\sigma}^{\circ} \cap N}\left(d+\kappa\left(n^{\prime}\right)-1\right) \\
& +\sum_{\sigma \in \Sigma^{\prime}(d)} \sum_{n^{\prime} \in \square_{\sigma}^{\circ} \cap N}\left(d+\kappa\left(n^{\prime}\right)\right)\left(d+\kappa\left(n^{\prime}\right)-1\right) .
\end{aligned}
$$

By Equation (13), we obtain the equality

$$
\begin{aligned}
& 2 \cdot \sum_{\sigma \in \Sigma^{\prime}(d-2)}\left|\square_{\sigma}^{\circ} \cap N\right|+2 \cdot \sum_{\sigma \in \Sigma^{\prime}(d-1)} \sum_{n^{\prime} \in \square_{\sigma}^{\circ} \cap N}\left(d+\kappa\left(n^{\prime}\right)-1\right) \\
& \quad+\sum_{\sigma \in \Sigma^{\prime}(d)} \sum_{n^{\prime} \in \square_{\sigma}^{\circ} \cap N}\left(d+\kappa\left(n^{\prime}\right)\right)\left(d+\kappa\left(n^{\prime}\right)-1\right)=\frac{\left(3 d^{2}-5 d\right)}{12} v(\Sigma)+\frac{1}{6} c_{1}(X) \cdot c_{d-1}^{s t r}(X)
\end{aligned}
$$

because $c_{d}^{s t r}(X)=v(\Sigma)$ by Corollary 3.3 (cf. [Bat98, Proposition 4.10]). Furthermore,

$$
c_{1}(X) \cdot c_{d-1}^{s t r}(X)=\left[-K_{X}\right] \cdot c_{d-1}^{s t r}(X)=\sum_{\sigma \in \Sigma(d-1)} v(\sigma) \cdot l_{-K_{X}}(\sigma)
$$


by Proposition 3.4 and

$$
c_{1}(X) \cdot c_{d-1}^{s t r}(X)=\left[-K_{X}\right] \cdot c_{d-1}^{s t r}(X)=\sum_{\sigma \in \Sigma(d-1)} v(\sigma) \cdot v\left(\Delta_{-K_{X}}^{\sigma}\right)
$$

by Theorem 3.5 if $-K_{X}$ is semiample.

Recall that a normal projective surface is called log del Pezzo surface if it has at worst log-terminal singularities and if its anticanonical divisor is an ample $\mathbb{Q}$-Cartier divisor. Toric log del Pezzo surfaces one-to-one correspond to convex lattice polygons $\Delta \subseteq N_{\mathbb{R}}$ containing the origin in its interior such that the vertices of $\Delta$ are primitive lattice points in $N$. These polygons $\Delta$ are called LDP-polygons [KKN10]. The fan $\Sigma$ defining a toric log del Pezzo surface $X$ consists of cones over faces of $\Delta$. In particular, any LDP-polygon $\Delta$ is the convex hull of all primitive lattice generators of 1-dimensional cones of $\Sigma(1)$. We remark that in general the vertices of the dual polygon $\Delta^{*} \subseteq M_{\mathbb{R}}$ are not lattice points in $M$.

We propose a new combinatorial identity that is equivalent to the stringy LibgoberWood identity (13) and relates the number 12 to LDP-polygons $\Delta$ :

Corollary 4.5. Let $X$ be a toric log del Pezzo surface defined by a fan $\Sigma$ in $N_{\mathbb{R}}$ together with the corresponding LDP-polygon $\Delta \subseteq N_{\mathbb{R}}$. Then

$$
v(\Delta)+v\left(\Delta^{*}\right)=12 \sum_{n \in \Delta \cap N}(\kappa(n)+1)^{2},
$$

where $\kappa$ the $\Sigma$-piecewise linear function corresponding to the anticanonical divisor of $X$. In particular, one always has $v(\Delta)+v\left(\Delta^{*}\right) \geq 12$ and equality holds if and only if $\Delta$ is a reflexive polygon.

Proof. We use the formula for the stringy $E$-function from Corollary 4.2 and obtain

$$
E_{s t r}(X ; u, 1)=(u-1)^{2}+\sum_{\substack{n \in N \\ \kappa(n)=-1}} u+\sum_{\substack{n \in N \\-1<\kappa(n)<0}}\left(u^{2+\kappa(n)}+u^{-\kappa(n)}\right) .
$$

Therefore,

$$
\begin{aligned}
\left.\frac{d^{2}}{d u^{2}} E_{s t r}(X ; u, 1)\right|_{u=1} & =2+\sum_{0 \neq n \in \Delta^{\circ} \cap N}((2+\kappa(n))(1+\kappa(n))+(-\kappa(n))(-\kappa(n)-1)) \\
& =2 \sum_{n \in \Delta^{\circ} \cap N}(\kappa(n)+1)^{2}=2 \sum_{n \in \Delta \cap N}(\kappa(n)+1)^{2},
\end{aligned}
$$

where $\Delta^{\circ}$ denotes the interior of the polygon $\Delta$. By Equation (13), we get the equality

$$
2 \sum_{n \in \Delta \cap N}(\kappa(n)+1)^{2}=\frac{1}{6} c_{2}^{s t r}(X)+\frac{1}{6} c_{1}(X)^{2}=\frac{1}{6}\left(v(\Delta)+v\left(\Delta^{*}\right)\right)
$$

because $c_{2}^{\text {str }}(X)=v(\Sigma)=v(\Delta)$ and $c_{1}(X)^{2}=v\left(\Delta^{*}\right)$. One has

$$
\sum_{n \in \Delta \cap N}(\kappa(n)+1)^{2} \geq 1
$$


because the origin is contained in $\Delta$. Equality holds, if and only if the origin is the unique interior lattice point $n$ in $\Delta$.

\section{Applications to Reflexive and Gorenstein polytopes}

Let $\Delta \subseteq N_{\mathbb{R}}$ be a $d$-dimensional convex lattice polytope that contains the origin in its interior. Denote by $\Sigma$ a fan in $N_{\mathbb{R}}$ consisting of cones over faces of $\Delta$ that defines a normal projective toric variety $X$. The polytope $\Delta$ is called reflexive if its dual $\Delta^{*}=\left\{y \in M_{\mathbb{R}} \mid\langle y, x\rangle \geq-1 \forall x \in \Delta\right\}$ is also a lattice polytope. If $\Delta$ is reflexive, then the associated variety $X$ is a Gorenstein toric Fano variety (i.e., $q_{X}=1$ ).

We are interested in a combinatorial identity for reflexive polytopes $\Delta$ that is equivalent to the stringy Libgober-Wood identity (14) for Gorenstein toric Fano varieties. For this purpose, we observe that the generalized stringy Hodge numbers $\psi_{\alpha}(\Sigma)$ in

$$
E_{s t r}(X ; u, v)=\sum_{\alpha \in[0, d] \cap \mathbb{Z}} \psi_{\alpha}(\Sigma)(u v)^{\alpha}
$$

are equal to the nonnegative integral coefficients $\psi_{d}(\Delta), \ldots, \psi_{0}(\Delta)$ in the numerator of the Ehrhart power series

$$
P_{\Delta}(t)=\frac{\psi_{d}(\Delta) t^{d}+\ldots+\psi_{1}(\Delta) t+\psi_{0}(\Delta)}{(1-t)^{d+1}} .
$$

Lemma 5.1. Let $\Delta \subseteq N_{\mathbb{R}}$ be a d-dimensional reflexive polytope and $X$ the associated Gorenstein toric Fano variety. Then

$$
E_{s t r}(X ; u, v)=\psi_{d}(\Delta)(u v)^{d}+\ldots+\psi_{1}(\Delta)(u v)+\psi_{0}(\Delta),
$$

i.e., $\psi_{\alpha}(\Sigma)=\psi_{\alpha}(\Delta)$ for all $\alpha \in[0, d] \cap \mathbb{Z}$, where $\psi_{\alpha}(\Sigma)$ and $\psi_{\alpha}(\Delta)$ are given as above.

Proof. By Equation (15), we have

$$
E_{s t r}(X ; u, v)=(u v-1)^{d} \sum_{n \in N}(u v)^{\kappa(n)}=(u v-1)^{d} \sum_{k \geq 0} \sum_{\substack{n \in N \\ \kappa(n)=-k}}(u v)^{-k},
$$

since the fan $\Sigma$ defining $X$ is complete. We note that the number of lattice points $n \in N$ such that $\kappa(n)=-k$ equals $|k \Delta \cap N|-|(k-1) \Delta \cap N|$. Therefore, we get

$$
E_{s t r}(X ; u, v)=(u v-1)^{d}\left(1-(u v)^{-1}\right) \sum_{k \geq 0}|k \Delta \cap N|(u v)^{-k} .
$$

Using the definition of $P_{\Delta}\left((u v)^{-1}\right)$ and Equation (16), this implies

$$
\begin{aligned}
E_{\text {str }}(X ; u, v) & =(u v)^{d}\left(1-(u v)^{-1}\right)^{d+1} \cdot P_{\Delta}\left((u v)^{-1}\right) \\
& =\psi_{d}(\Delta)+\ldots+\psi_{1}(\Delta)(u v)^{d-1}+\psi_{0}(\Delta)(u v)^{d} \\
& =\psi_{d}(\Delta)(u v)^{d}+\ldots+\psi_{1}(\Delta)(u v)+\psi_{0}(\Delta)
\end{aligned}
$$

because $\psi_{\alpha}(\Delta)=\psi_{d-\alpha}(\Delta)$ for all $0 \leq \alpha \leq d$ [Bat93, Theorem 2.11]. 
Let $\Delta \subseteq N_{\mathbb{R}}$ be a $d$-dimensional convex lattice polytope that contains the origin in its interior. If $\theta$ is a face of $\Delta$, then the face $\theta^{*}=\left\{y \in \Delta^{*} \mid\langle y, x\rangle=-1 \forall x \in \theta\right\} \subseteq \Delta^{*}$ is the dual face to $\theta$. This establishes a one-to-one order-reversing correspondence between faces of $\Delta$ and faces of $\Delta^{*}$ such that $\operatorname{dim}(\theta)+\operatorname{dim}\left(\theta^{*}\right)=d-1$.

Theorem 5.2. Let $\Delta \subseteq N_{\mathbb{R}}$ be a d-dimensional reflexive polytope. Then the stringy Libgober-Wood identity for the Gorenstein toric Fano variety $X$ corresponding to $\Delta$ is equivalent to

$$
\sum_{\alpha \in[0, d] \cap \mathbb{Z}} \psi_{\alpha}(\Delta)\left(\alpha-\frac{d}{2}\right)^{2}=\frac{d}{12} v(\Delta)+\frac{1}{6} \sum_{\substack{\theta \preceq \Delta \\ \operatorname{dim}(\theta)=d-2}} v(\theta) \cdot v\left(\theta^{*}\right),
$$

where $\psi_{\alpha}(\Delta)$ are the coefficients in the numerator of the Ehrhart power series $P_{\Delta}(t)$. Proof. Using Lemma 5.1 and Theorem 4.3, we obtain

$$
\sum_{\alpha \in[0, d] \cap \mathbb{Z}} \psi_{\alpha}(\Delta)\left(\alpha-\frac{d}{2}\right)^{2}=\frac{d}{12} v(\Sigma)+\frac{1}{6} \sum_{\sigma \in \Sigma(d-1)} v(\sigma) \cdot v\left(\Delta_{-K_{X}}^{\sigma}\right),
$$

since the anticanonical divisor $-K_{X}$ is ample. It remains to use $v(\Sigma)=v(\Delta)$ and

$$
\sum_{\sigma \in \Sigma(d-1)} v(\sigma) \cdot v\left(\Delta_{-K_{X}}^{\sigma}\right)=\sum_{\substack{\theta \leq \Delta, \operatorname{dim}(\bar{\theta})=d-2}} v(\theta) \cdot v\left(\theta^{*}\right)
$$

since $\Delta_{-K_{X}}=\Delta^{*}, \sigma$ is a cone over a face $\theta$ of $\Delta, \Delta_{-K_{X}}^{\sigma}=\theta^{*}$, and every facet of $\Delta$ has lattice distance 1 to the origin.

The well-known identities for reflexive polytopes of dimension 2 and 3 follow from the above statement:

Corollary 5.3. Let $\Delta \subseteq N_{\mathbb{R}}$ be a 2-dimensional reflexive polytope. Then the stringy Libgober-Wood identity is equivalent to

$$
v(\Delta)+v\left(\Delta^{*}\right)=12 .
$$

Proof. Using Theorem 5.2, we get

$$
\sum_{\alpha \in[0,2] \cap \mathbb{Z}} \psi_{\alpha}(\Delta)(\alpha-1)^{2}=\frac{1}{6} v(\Delta)+\frac{1}{6} \sum_{\substack{\theta \leq \Delta \\ \operatorname{dim}(\theta)=0}} v(\theta) \cdot v\left(\theta^{*}\right) .
$$

Moreover, $\sum_{\alpha \in[0,2] \cap \mathbb{Z}} \psi_{\alpha}(\Delta)(\alpha-1)^{2}=2$ because $\psi_{0}(\Delta)=\psi_{2}(\Delta)=1$. It remains to apply the equalities

$$
\sum_{\substack{\theta \leq \Delta \\ \operatorname{dim}(\theta)=0}} v(\theta) \cdot v\left(\theta^{*}\right)=\sum_{\substack{\theta \leq \Delta \\ \operatorname{dim}(\theta)=0}} v\left(\theta^{*}\right)=v\left(\Delta^{*}\right)
$$

that hold because $\Delta^{*}$ is reflexive and $v(\theta)=1$ if $\operatorname{dim}(\theta)=0$. 
Corollary 5.4. Let $\Delta \subseteq N_{\mathbb{R}}$ be a 3-dimensional reflexive polytope. Then the stringy Libgober-Wood identity is equivalent to

$$
\sum_{\substack{\theta \preceq \Delta \\ \operatorname{dim}(\theta)=1}} v(\theta) \cdot v\left(\theta^{*}\right)=24
$$

Proof. Theorem 5.2 implies

$$
\sum_{\alpha \in[0,3] \cap \mathbb{Z}} \psi_{\alpha}(\Delta)\left(\alpha-\frac{3}{2}\right)^{2}=\frac{1}{4} v(\Delta)+\frac{1}{6} \sum_{\substack{\theta \leq \Delta \\ \operatorname{dim}(\theta)=1}} v(\theta) \cdot v\left(\theta^{*}\right) .
$$

The coefficients $\psi_{\alpha}(\Delta)$ in the numerator of the Ehrhart power series $P_{\Delta}(t)$ are $\psi_{0}(\Delta)=\psi_{3}(\Delta)=1$ and $\psi_{1}(\Delta)=\psi_{2}(\Delta)=|\Delta \cap N|-4$ [Bat93, Theorem 2.11], i.e.,

$$
\sum_{\alpha \in[0,3] \cap \mathbb{Z}} \psi_{\alpha}(\Delta)\left(\alpha-\frac{3}{2}\right)^{2}=\frac{9}{2}+\frac{1}{2} \psi_{1}(\Delta)
$$

and we conclude

$$
\sum_{\substack{\theta \leq \Delta \\ \operatorname{dim}(\theta)=1}} v(\theta) \cdot v\left(\theta^{*}\right)=27+3\left(\psi_{1}(\Delta)-\frac{1}{2} v(\Delta)\right)=24,
$$

where the last equality holds because $v(\Delta)=v(\Sigma)=e_{s t r}(X)=\sum_{\alpha \in[0,3] \cap \mathbb{Z}} \psi_{\alpha}(\Delta)$.

If the dimension $d$ of a reflexive polytope is greater than 3, the identity in Theorem 5.2 is not anymore a symmetric equation with respect to polar duality between $\Delta$ and $\Delta^{*}$. The received identities for reflexive polytopes $\Delta$ and $\Delta^{*}$ of dimension $\geq 4$ are not equivalent to each other. The latter is easy to see in the case $d=4$ :

Corollary 5.5. Let $\Delta \subseteq N_{\mathbb{R}}$ be a 4-dimensional reflexive polytope. Then the stringy Libgober-Wood indentity is equivalent to

$$
12 \cdot|\partial \Delta \cap N|=2 \cdot v(\Delta)+\sum_{\substack{\theta \leq \Delta \\ \operatorname{dim}(\theta)=2}} v(\theta) \cdot v\left(\theta^{*}\right),
$$

where $\partial \Delta$ denotes the boundary of $\Delta$ and $|\partial \Delta \cap N|$ the number of lattice points in $\partial \Delta$.

Proof. By Theorem 5.2, we have

$$
\sum_{\alpha \in[0,4] \cap \mathbb{Z}} \psi_{\alpha}(\Delta)(\alpha-2)^{2}=\frac{1}{3} v(\Delta)+\frac{1}{6} \sum_{\substack{\theta \leq \Delta \\ \operatorname{dim}(\theta)=2}} v(\theta) \cdot v\left(\theta^{*}\right) .
$$

Furthermore, $\psi_{0}(\Delta)=\psi_{4}(\Delta)=1$ and $\psi_{1}(\Delta)=\psi_{3}(\Delta)=|\Delta \cap N|-5$ [Bat93, Theorem 2.11], i.e., we obtain

$$
\sum_{\alpha \in[0,4] \cap \mathbb{Z}} \psi_{\alpha}(\Delta)(\alpha-2)^{2}=8+2 \cdot(|\Delta \cap N|-5)
$$


It remains to apply $|\partial \Delta \cap N|=|\Delta \cap N|-1$ because a reflexive polytope $\Delta$ has a single interior lattice point.

One may produce a more "mirror symmetric" identity for arbitrary 4-dimensional reflexive polytopes by summing the equations from Corollary 5.5 for $\Delta$ and $\Delta^{*}$.

Corollary 5.6. Let $\Delta \subseteq N_{\mathbb{R}}$ be a 4-dimensional reflexive polytope. Then

$$
12 \cdot\left(|\partial \Delta \cap N|+\left|\partial \Delta^{*} \cap M\right|\right)=2 \cdot\left(v(\Delta)+v\left(\Delta^{*}\right)\right)+\sum_{\substack{\theta \leq \Delta \\ \operatorname{dim}(\bar{\theta})=1,2}} v(\theta) \cdot v\left(\theta^{*}\right) .
$$

Let $r$ be a positive integer. A $d$-dimensional lattice polytope $\Delta \subseteq M_{\mathbb{R}}$ is called Gorenstein polytope of index $r$ if $r \Delta-m$ is a reflexive polytope for some lattice point $m \in M$. Note that reflexive polytopes are Gorenstein polytopes of index $r=1$. Denote by $\Sigma$ the normal fan in $N_{\mathbb{R}}$ of the polytope $\Delta$ (or, equivalently, of $r \Delta$ ). The fan $\Sigma$ defines a Gorenstein toric Fano variety $X$ such that its anticanonical class $c_{1}(X)$ is divisible by $r$ in $\operatorname{Pic}(X)$.

There exists a duality for Gorenstein polytopes that generalizes the polar duality for reflexive polytopes. For this purpose, we associate to every $d$-dimensional Gorenstein polytope $\Delta \subseteq M_{\mathbb{R}}$ of index $r$ the $(d+1)$-dimensional cone

$$
C_{\Delta}:=\left\{(y, \lambda) \in M_{\mathbb{R}} \oplus \mathbb{R} \mid y \in \lambda \Delta\right\} \subseteq M_{\mathbb{R}} \oplus \mathbb{R} .
$$

The dual cone $C_{\Delta}^{\vee} \subseteq N_{\mathbb{R}} \oplus \mathbb{R}$ is defined as

$$
C_{\Delta}^{\vee}:=\left\{(x, \mu) \in N_{\mathbb{R}} \oplus \mathbb{R} \mid\langle y, x\rangle+\lambda \mu \geq 0 \forall(y, \lambda) \in C_{\Delta}\right\}
$$

and the $l$-th slice $C_{\Delta}^{\vee}(l)$ of $C_{\Delta}^{\vee}$ is defined as the lattice polytope

$$
C_{\Delta}^{\vee}(l):=C_{\Delta}^{\vee} \cap\left\{(x, \mu) \in N_{\mathbb{R}} \oplus \mathbb{R} \mid\langle m, x\rangle+r \mu=l\right\} \subseteq N_{\mathbb{R}} \oplus \mathbb{R} .
$$

The lattice polytope $\Delta^{*}:=C_{\Delta}^{\vee}(1)$ is again a Gorenstein polytope of index $r$ and is called dual Gorenstein polytope to $\Delta$. The duality between two $(d+1)$-dimensional cones $C_{\Delta}$ and $C_{\Delta}^{\vee}$ establishes a one-to-one order-reversing correspondence between faces of $C_{\Delta}$ and $C_{\Delta}^{\vee}$ that induces a duality between faces of the Gorenstein polytopes $\Delta$ and $\Delta^{*}=C_{\Delta}^{\vee}(1)$. It is important to note that the reflexive polytope $(r \Delta)^{*}$ and the Gorenstein polytope $\Delta^{*}$ are not only naturally combinatorially isomorphic, but this isomorphism also induces isomorphisms between proper faces of $(r \Delta)^{*}$ and $\Delta^{*}$ considered as lattice polytopes BN08].

The fan $\Sigma$ in $N_{\mathbb{R}}$ can be constructed via the projection

$$
N_{\mathbb{R}} \oplus \mathbb{R} \rightarrow\left(N_{\mathbb{R}} \oplus \mathbb{R}\right) / \mathbb{R}(n, r) \cong N_{\mathbb{R}}
$$

of all proper faces of the cone $C_{\Delta}^{\vee}$ along the 1-dimensional subspace generated by the unique interior lattice point $(n, r)$ in the reflexive polytope $C_{\Delta}^{\vee}(r)$.

It is well-known [BD96, Corollary 7.10] that the stringy Euler number of a generic Calabi-Yau hypersurface $Z$ in a Gorenstein toric Fano variety $X$ can be computed 
via volumes of faces $\theta \preceq \Delta$ and $\theta^{*} \preceq \Delta^{*}$ of $d$-dimensional reflexive polytopes $\Delta$ respectively $\Delta^{*}$ as

$$
e_{s t r}(Z)=c_{d-1}^{s t r}(Z)=\sum_{k=0}^{d-3}(-1)^{k} \sum_{\substack{\theta \leq \Delta \\ \operatorname{dim}(\bar{\theta})=k+1}} v(\theta) \cdot v\left(\theta^{*}\right) .
$$

Using our previous results and the duality between faces $\theta \preceq \Delta$ and $\theta^{*} \preceq \Delta^{*}$ of Gorenstein polytopes $\Delta$ respectively $\Delta^{*}$, we can generalize this combinatorial formula to the case of generic Calabi-Yau complete intersections in Gorenstein toric Fano varieties.

Theorem 5.7. Let $X$ be a Gorenstein toric Fano variety associated with a $d$-dimensional Gorenstein polytope $\Delta \subseteq M_{\mathbb{R}}$ of index $r$ and $D$ an ample torus-invariant Cartier divisor on $X$ such that $[D]=\frac{1}{r} c_{1}(X)$. Denote by $Z_{1}, \ldots, Z_{r} \subseteq X$ generic semiample Cartier divisors such that $\left[Z_{1}\right]=\ldots=\left[Z_{r}\right]=[D]=\frac{1}{r} c_{1}(X)$. Then the stringy Euler number of the Calabi-Yau complete intersection $S:=Z_{1} \cap \ldots \cap Z_{r}$ is

$$
c_{d-r}^{s t r}(S)=\sum_{k=0}^{d-r-1}(-1)^{k}\left(\begin{array}{c}
k+r-1 \\
r-1
\end{array}\right) \sum_{\substack{\theta \leq \Delta \\
\operatorname{dim}(\theta)=k+r}} v(\theta) \cdot v\left(\theta^{*}\right)+(-1)^{d-r}\left(\begin{array}{l}
d-1 \\
r-1
\end{array}\right) v(\Delta) .
$$

Proof. By Corollary 3.7, we have

$$
c_{d-r}^{s t r}(S)=\sum_{k=0}^{d-r}(-1)^{k}\left(\begin{array}{c}
k+r-1 \\
r-1
\end{array}\right) \sum_{\sigma \in \Sigma(d-r-k)} v(\sigma) \cdot v\left(\Delta_{D}^{\sigma}\right),
$$

where $\Sigma$ is the associated fan to $X$ and $\Delta_{D}^{\sigma}$ a face of the lattice polytope $\Delta_{D}$. Let $\sigma$ be a $(d-r-k)$-dimensional cone of $\Sigma(0 \leq k \leq d-r-1)$. Then $\sigma$ can be considered as a cone over a $(d-r-k-1)$-dimensional proper face of the reflexive polytope $(r \Delta)^{*}$, which we naturally identify with the corresponding proper face $\theta^{*} \preceq \Delta^{*}$ of the dual Gorenstein polytope $\Delta^{*}[\mathrm{BN08}$, Proposition 1.16]. Therefore, we obtain $v(\sigma)=v\left(\theta^{*}\right)$. On the other hand, the lattice polytope $\Delta_{D}$ is exactly the Gorenstein polytope $\Delta$ and $\theta^{*}$ is the dual face to a $(k+r)$-dimensional face $\Delta_{D}^{\sigma}=\theta$ of $\Delta$. This implies

$$
\sum_{\sigma \in \Sigma(d-r-k)} v(\sigma) \cdot v\left(\Delta_{D}^{\sigma}\right)=\sum_{\substack{\theta^{*} \preceq \Delta^{*} \\ \operatorname{dim}\left(\theta^{*}\right)=d-k-r-1}} v\left(\theta^{*}\right) \cdot v(\theta)=\sum_{\substack{\theta \preceq \Delta \\ \operatorname{dim}(\bar{\theta})=k+r}} v(\theta) \cdot v\left(\theta^{*}\right)
$$

for all $0 \leq k \leq d-r-1$. It remains to note that in the case $k=d-r$ (i.e., $\operatorname{dim}(\sigma)=0)$, one has $\Delta_{D}^{\sigma}=\Delta, v\left(\Delta_{D}^{\sigma}\right)=v(\Delta)$, and $v(\sigma)=1$.

The combinatorial formula from Corollary 5.4

$$
24=\sum_{\substack{\theta \preceq \Delta \\ \operatorname{dim}(\theta)=1}} v(\theta) \cdot v\left(\theta^{*}\right)
$$

for a 3 -dimensional reflexive polytope $\Delta$ can be generalized for arbitrary $d$-dimensional Gorenstein polytopes $\Delta(d \geq 3)$ of index $r=d-2$. 
Proposition 5.8. Let $\Delta \subseteq M_{\mathbb{R}}$ be a d-dimensional Gorenstein polytope of index $r=d-2$. Then

$$
24=\sum_{\substack{\theta \preceq \Delta \\ \operatorname{dim}(\theta)=r}} v(\theta) \cdot v\left(\theta^{*}\right)+\frac{r(1-r)}{2} v(\Delta) .
$$

Proof. Let $S:=Z_{1} \cap \ldots \cap Z_{r}$ be a generic Calabi-Yau complete intersection in the Gorenstein toric Fano variety $X$ associated to $\Delta$, i.e., $Z_{1}, \ldots, Z_{r}$ are $r$ generic ample Cartier divisors on $X$ such that $\left[Z_{1}\right]=\ldots=\left[Z_{r}\right]=\frac{1}{r} c_{1}(X)$. Then $\operatorname{dim}(S)=2$, i.e., $S$ is a (possibly singular) $K 3$-surface. The stringy Euler number $c_{2}^{\text {str }}(S)$ of $S$ equals the usual Euler number $c_{2}(\widetilde{S})$ of the minimal (crepant) desingularization $\widetilde{S}$ of $S$. Since $\widetilde{S}$ is a smooth $K 3$-surface, we have $c_{2}^{\text {str }}(S)=c_{2}(\widetilde{S})=24$. Using Theorem 5.7 , we obtain

$$
24=c_{2}^{s t r}(S)=\sum_{\substack{\theta \leq \Delta \\ \operatorname{dim}(\theta)=r}} v(\theta) \cdot v\left(\theta^{*}\right)-r \cdot \sum_{\substack{\theta \leq \Delta \\ \operatorname{dim}(\theta)=d-1}} v(\theta) \cdot v\left(\theta^{*}\right)+\frac{(r+1) r}{2} v(\Delta) .
$$

Since $r \Delta$ is a reflexive polytope, one has

$$
r^{d} v(\Delta)=v(r \Delta)=\sum_{\substack{r \theta \preceq r \Delta \\ \operatorname{dim}(r \bar{\theta})=d-1}} v(r \theta)=r^{d-1} \sum_{\substack{\theta \preceq \Delta \\ \operatorname{dim}(\bar{\theta})=d-1}} v(\theta) .
$$

Moreover, $v\left(\theta^{*}\right)=1$ if $\operatorname{dim}\left(\theta^{*}\right)=0$. It remains to apply the equalities

$$
\sum_{\substack{\theta \preceq \Delta \\ \operatorname{dim}(\theta)=d-1}} v(\theta) \cdot v\left(\theta^{*}\right)=\sum_{\substack{\theta \preceq \Delta \\ \operatorname{dim}(\theta)=d-1}} v(\theta)=r v(\Delta) .
$$

It was proved in BJ10, Proposition 3.4] that the combinatorial identity

$$
12=\sum_{\substack{\theta \preceq \Delta \\ \operatorname{dim}(\theta)=1}} v(\theta) \cdot v\left(\theta^{*}\right)
$$

holds for any 3 -dimensional Gorenstein polytope $\Delta$ of index 2 . We show that this identity can be generalized for arbitrary $d$-dimensional Gorenstein polytopes $\Delta(d \geq$ 3) of index $r=d-1$.

Proposition 5.9. Let $\Delta \subseteq M_{\mathbb{R}}$ be a d-dimensional Gorenstein polytope of index $r=d-1$. Then

$$
12=\sum_{\substack{\theta \leq \Delta \\ \operatorname{dim}(\bar{\theta})=r-1}} v(\theta) \cdot v\left(\theta^{*}\right)+\frac{r(1-r)+2}{2} v(\Delta) .
$$

Proof. Let $S:=Z_{1} \cap \ldots \cap Z_{r-1}$ be a generic complete intersection in the Gorenstein toric Fano variety $X$ associated to $\Delta$, i.e., $Z_{1}, \ldots, Z_{r-1}$ are $r-1$ generic ample Cartier divisors on $X$ such that $[D]:=\left[Z_{1}\right]=\ldots=\left[Z_{r-1}\right]=\frac{1}{r} c_{1}(X)$. Then $\operatorname{dim}(S)=2$, i.e., $S$ is a (possibly singular) del Pezzo surface. The stringy Euler number $c_{2}^{\text {str }}(S)$ of $S$ equals the usual Euler number $c_{2}(\widetilde{S})$ of the minimal (crepant) desingularization $\widetilde{S}$ 
of $S$. Then $\widetilde{S}$ is a smooth rational surface and we have $c_{2}^{\text {str }}(S)=c_{2}(\widetilde{S})$ respectively $c_{1}(S)^{2}=c_{1}(\widetilde{S})^{2}$. Using Noether's Theorem for $\widetilde{S}$, we obtain

$$
12=c_{2}(\widetilde{S})+c_{1}(\widetilde{S})^{2}=c_{2}^{s t r}(S)+c_{1}(S)^{2} .
$$

It remains to derive combinatorial formulas for $c_{1}(S)^{2}$ and $c_{2}^{\text {str }}(S)$. By the adjunction formula, the anticanonical class of $S$ is the restriction of $c_{1}(X)-(r-1)[D]=[D]$ to $S$. Therefore, $c_{1}(S)^{2}=[D]^{2} \cdot[D]^{r-1}=[D]^{d}=v(\Delta)$. By Corollary 3.7, we have

$$
c_{2}^{s t r}(S)=\sum_{\sigma \in \Sigma(2)} v(\sigma) \cdot v\left(\Delta_{D}^{\sigma}\right)-(r-1) \cdot \sum_{\sigma \in \Sigma(1)} v(\sigma) \cdot v\left(\Delta_{D}^{\sigma}\right)+\frac{r(r-1)}{2} v\left(\Delta_{D}\right) .
$$

Using the same arguments as in the proof of Theorem 5.7, we can rewrite the above equation as

$$
c_{2}^{\text {str }}(S)=\sum_{\substack{\theta \leq \Delta \\ \operatorname{dim}(\theta)=d-2}} v(\theta) \cdot v\left(\theta^{*}\right)-(r-1) \cdot \sum_{\substack{\theta \leq \Delta \\ \operatorname{dim}(\theta)=d-1}} v(\theta) \cdot v\left(\theta^{*}\right)+\frac{r(r-1)}{2} v(\Delta) .
$$

Using the last equation

$$
\sum_{\substack{\theta \leq \Delta \\ \operatorname{dim}(\bar{\theta})=d-1}} v(\theta) \cdot v\left(\theta^{*}\right)=r v(\Delta)
$$

in the proof of Proposition 5.8, we obtain

$$
c_{2}^{\text {str }}(S)=\sum_{\substack{\theta \subseteq \Delta \\ \operatorname{dim}(\bar{\theta})=r-1}} v(\theta) \cdot v\left(\theta^{*}\right)+\frac{r(1-r)}{2} v(\Delta) .
$$

\section{REFERENCES}

[Alu04] P. Aluffi, Chern classes of birational varieties, International Mathematics Research Notices (2004), no. 63, 33673377. 1

[Alu05] _ Modification systems and integration in their Chow groups, Selecta Mathematica 11 (2005), 155-202. 1, 3

[Bat93] V. V. Batyrev, Variations of the mixed Hodge structure of affine hypersurfaces in algebraic tori, Duke Math. J. 69 (1993), no. 2, 349-409. 6, 18, 23, 25

[Bat94] _ Dual Polyhedra and Mirror Symmetry for Calabi-Yau Hypersurfaces in Toric Varieties, Journal of Algebraic Geometry 3 (1994), no. 3, 493-535. 2, 5

[Bat98] _ Stringy Hodge numbers of varieties with Gorenstein canonical singularities, Integrable systems and algebraic geometry (Kobe/Kyoto 1997), World Sci. Publ., River Edge, NJ (1998), 1-31. 1, 2, 11, 12, 17, 18, 20, 21

[Bat00] _ Stringy Hodge numbers and Virasoro algebra, Math. Res. Lett. 7 (2000), no. 12, 155-164. 2, 4, 11, 17, 20

[BB97] V. V. Batyrev and L. A. Borisov, Dual cones and mirror symmetry for generalized Calabi-Yau manifolds, Mirror Symmetry II, AMS/IP Stud. Adv. Math. 1, Amer. Math. Soc., Providence, RI (1997), 71-86. 2, 5

[BD96] V. V. Batyrev and D. I. Dais, Strong McKay correspondende, string-theoretic Hodge numbers and mirror symmetry, Topology 35 (1996), no. 4, 901-929. 1, 2, 5, 26 
[BJ10] V. V. Batyrev and D. Juny, Classification of Gorenstein toric del Pezzo varieties in arbitrary dimension, Mosc. Math. J. 10 (2010), no. 2, 285-316. 28

[BN08] V. V. Batyrev and B. Nill, Combinatorial aspects of mirror symmetry, Integer points in polyhedra-geometry, number theory, representation theory, algebra, optimization, statistics; Contemp. Math., Amer. Math. Soc., Providence, RI 452 (2008), 35-66. 26. 27

[Bor97] L. A. Borisov, On Betti numbers and Chern classes of varieties with trivial odd cohomology groups, arXiv:alg-geom/9703023, 1997. 16

[CLS11] D. A. Cox, J. B. Little, and H. K. Schenck, Toric Varieties, American Mathematical Society, 2011. 14, 15

[dFLNU07] T. de Fernex, E. Lupercio, T. Nevins, and B. Uribe, Stringy Chern classes of singular varieties, Advances in Mathematics 208 (2007), 597-621. 1, 3, 6, 7

[DHVW85] L. Dixon, J. A. Harvey, C. Vafa, and E. Witten, Strings on orbifolds, Nuclear Physics B 261 (1985), 678-686. 1

[Dou16] A. Douai, Global spectra, polytopes and stacky invariants, arXiv:1603.08693, 2016. 2

[Ful93] W. Fulton, Introduction to Toric Varieties, Princeton University Press, 1993. 16

[Ful98] _ Intersection Theory, Springer, 1998. 8

[GvHS16] L. Godinho, F. von Heymann, and S. Sabatini, 12, 24 and Beyond, arXiv:1604.00277, 2016. 2

[HH90] F. Hirzebruch and T. Höfer, On the Euler number of an orbifold, Math. Ann. 286 (1990), no. 1-3, 255-260. 1

[KKN10] A. M. Kasprzyk, M. Kreuzer, and B. Nill, On the combinatorial classification of toric log del Pezzo surfaces, LMS J. Comput. Math. 13 (2010), 33-46. 5, 22

[LW90] A. S. Libgober and J. W. Wood, Uniqueness of the complex structure on Kähler manifolds of certain homotopy types, J. Differential Geom. 32 (1990), no. 1, 139-154. 2, 16

[Sal96] S. M. Salamon, Cohomology of Kähler manifolds with $c_{1}=0$, Manifolds and geometry (Pisa, 1993), Sympos. Math., XXXVI, Cambridge Univ. Press (1996), 294-310. 2

Mathematisches Institut, Universität Tübingen, Auf der Morgenstelle 10, 72076 TüBIngen, Germany

E-mail address: victor.batyrev@uni-tuebingen.de

Mathematisches Institut, Universität Tübingen, Auf Der Morgenstelle 10, 72076 TüBInGEn, GERMANy

E-mail address: karin.schaller@uni-tuebingen.de 